\title{
V-ATPase is a universal regulator of LC3 associated phagocytosis and non-canonical autophagy
}

Kirsty M. Hooper ${ }^{1}$, Elise Jacquin ${ }^{1,2}$, Taoyingnan $\mathrm{Li}^{3,4}$, Jonathan M. Goodwin ${ }^{5}$, John H. Brumell $^{3,4,6,7}$, Joanne Durgan ${ }^{1}$, Oliver Florey ${ }^{1}$

\author{
Affiliations \\ ${ }^{1}$ Signalling Programme, Babraham Institute, Cambridge, CB22 3AT, UK \\ ${ }^{2}$ INSERM UMR-S 1193, Université Paris-Saclay, Châtenay-Malabry, France \\ ${ }^{3}$ Department of Molecular Genetics, University of Toronto, Toronto, ON M5S 1A8, Canada \\ ${ }^{4}$ Cell Biology Program, Hospital for Sick Children, Toronto, ON, M5G 0A4, Canada \\ ${ }^{5}$ Casma Therapeutics, Cambridge, MA, 02139, USA \\ ${ }^{6}$ Institute of Medical Science, University of Toronto, Toronto, ON, M5S 1A8, Canada \\ ${ }^{7}$ SickKids IBD Centre, Hospital for Sick Children, Toronto, ON, M5G 0A4, Canada \\ Corresponding author: oliver.florey@babraham.ac.uk
}

\section{Running title:}

26 V-ATPase regulates non-canonical autophagy 


\section{Abstract}

29 Non-canonical autophagy is a key cellular pathway in immunity, cancer and

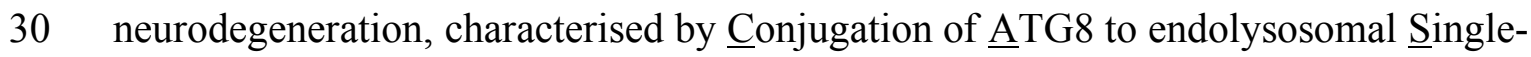

31 Membranes (CASM). CASM is activated by engulfment (endocytosis, phagocytosis),

32 agonists (STING, TRPML1) and infection (influenza), dependent on the ATG16L1 WD40-

33 domain, and specifically K490. However, the factor(s) associated with non-canonical

34 ATG16L1 recruitment, and CASM induction, remain unknown. Here, we investigate a role

35 for V-ATPase during non-canonical autophagy. We report that increased V0-V1 engagement

36 is associated with, and sufficient for, CASM activation. Upon V0-V1 binding, V-ATPase

37 directly recruits ATG16L1, via K490, during LC3-associated phagocytosis (LAP), STING-

38 and drug-induced CASM, indicating a common mechanism. Furthermore, during LAP, key

39 molecular players, including NADPH oxidase/ROS, converge on V-ATPase. Finally, we

40 show that LAP is sensitive to Salmonella SopF, which disrupts the V-ATPase-ATG16L1

41 axis, and provide evidence that CASM contributes to the Salmonella host response. Together,

42 these data identify V-ATPase as a universal regulator of CASM, and indicate that SopF

43 evolved in part to evade non-canonical autophagy. 


\section{Introduction}

45

Autophagy is a fundamental degradative process required for nutrient recycling, clearance of damaged organelles and pathogen responses. During autophagy, a collection of ATG proteins induce the formation and maturation of autophagosomes, which enwrap target cargo destined for lysosomal degradation (Choi et al., 2013). A subset of ATG proteins also act in parallel to mediate 'non-canonical autophagy', a pathway targeting endolysosomal compartments instead (Florey and Overholtzer, 2012; Heckmann et al., 2017). A diverse set of stimuli and cellular processes induce non-canonical autophagy, including stimulation of the TRPML1 calcium channel (Goodwin et al., 2021), activation of the STING pathway (Fischer et al., 2020), and disruption of endolysosomal ion balance, by pharmacological agents, Helicobacter pylori VacA toxin, or infection with influenza A virus (IAV) (Fletcher et al., 2018; Florey et al., 2015; Jacquin et al., 2017). Non-canonical autophagy is also associated with endocytic and engulfment processes, including entosis (Florey et al., 2011), LC3associated endocytosis (LANDO) (Heckmann et al., 2019) and LC3-associated phagocytosis (LAP) (Martinez et al., 2015; Sanjuan et al., 2007), with essential functions in the killing and clearance of pathogens (Gluschko et al., 2018; Hubber et al., 2017; Martinez et al., 2015), antigen presentation (Fletcher et al., 2018; Ma et al., 2012; Romao et al., 2013), clearance of and cytokine responses to apoptotic cells (Cunha et al., 2018; Florey et al., 2011; Martinez et al., 2011; Martinez et al., 2016; Martinez et al., 2015), clearance of accumulated $\beta$-amyloid (Heckmann et al., 2020; Heckmann et al., 2019), vision (Kim et al., 2013), response to influenza infection (Fletcher et al., 2018; Wang et al., 2021) and lysosome biogenesis (Goodwin et al., 2021). While the functional importance of non-canonical autophagy is clear, the mechanisms underlying the regulation and function of this pathway remain incompletely understood.

The canonical and non-canonical autophagy pathways share overlapping machineries, but with important differences. The non-canonical pathway is independent of the upstream autophagy regulators ATG9, mTor and the ULK1 initiation complex (ULK1/2, FIP200, ATG13 and ATG101) (Florey et al., 2011; Martinez et al., 2015). However, both pathways harness the core ATG8 lipidation machinery (ATG3, 4, 5, 7, 10, 12, 16L1). During canonical autophagy, ATG8s are conjugated exclusively to phosphatidylethanolamine (PE), at double membrane autophagosomes (Ichimura et al., 2000). In non-canonical autophagy, ATG8s are 
conjugated instead to single membrane, endolysosomal compartments, such as phagosomes,

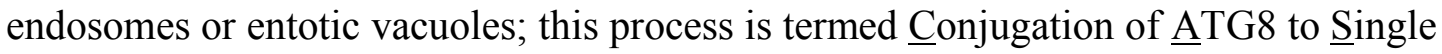
Membranes (CASM) and involves alternative conjugation to both phosphatidylserine (PS) and PE (Durgan et al., 2021).

ATG16L1 acts as a critical molecular hub, directing canonical and non-canonical autophagy at different sites, via different domains. During canonical autophagy, ATG16L1 is recruited to forming autophagosomes though its coiled coil domain (CCD), which interacts with WIPI2 and FIP200, thereby specifying the site of ATG8 conjugation (Dooley et al., 2014; Gammoh et al., 2013). During non-canonical autophagy, ATG16L1 is recruited instead to preformed endolysosomal membranes to drive CASM. The precise molecular mechanisms underlying this alternative recruitment remain unclear, but the ATG16L1 WD40 C-terminal domain (CTD), and certain ATG16L1 lipid binding motifs, are indispensable (Fletcher et al., 2018; Lystad et al., 2019; Rai et al., 2019). A single point mutation in ATG16L1, K490A, renders cells competent for canonical autophagy, but deficient in non-canonical autophagy, providing a highly specific genetic system to dissect these closely related pathways (Fletcher et al., 2018; Goodwin et al., 2021; Lystad et al., 2019).

The molecular mechanisms of non-canonical autophagy are best studied in the context of LC3-associated phagocytosis (LAP). LAP is dependent on Rubicon/Vps34, which mediates PI3P formation, and NADPH oxidase, which drives the generation of reactive oxygen species (ROS) (Huang et al., 2009; Martinez et al., 2015). However, quite how ROS production links mechanistically to the activation of LAP remains a key, unanswered question in the field. In addition, while LAP is dependent on Rubicon and ROS, other non-canonical autophagy processes, such as entosis and drug-induced CASM, are not (De Faveri et al., 2020; Florey et al., 2015; Jacquin et al., 2017), suggesting these molecular players are stimulus-specific inputs, rather than being universally required for CASM. This raises another important, open question regarding a molecular mechanism which might unify the diverse forms of noncanonical autophagy.

In considering a possible universal regulator for non-canonical autophagy, we turned our attention to the V-ATPase as a candidate. V-ATPase is a multi-subunit protein complex that acts as a molecular pump, generating proton gradients within intracellular compartments. It is required to acidify lysosomes, and supports the degradation of autophagosomes and engulfed 
111 material. As such, V-ATPase is generally thought to play a terminal, degradative role in

112 autophagy related processes. However, in the context of non-canonical autophagy, we

113 hypothesized that V-ATPase might also play an upstream, activating role, based on several

114 lines of evidence. Firstly, inhibition of V-ATPase by BafA1 blocks CASM induced by LAP,

115 entosis, ionophore and lysosomotropic drug stimulation, TRPML1 activation and STING

116 agonists (Fischer et al., 2020; Florey et al., 2015; Goodwin et al., 2021; Jacquin et al., 2017),

117 indicating a broad but undefined role in non-canonical autophagy. Secondly, a direct

118 interaction has recently been identified between V-ATPase and ATG16L1, during Salmonella

119 infection, which can be inhibited by the effector protein SopF (Xu et al., 2019). While this

120 interaction has been defined in the context of xenophagy, it is striking that it depends on the

121 ATG16L1 WD40 CTD, a domain specifically required for non-canonical autophagy, thus

122 hinting at a possible connection to CASM. Finally, and in line with this reasoning, STING

123 activation induces non-canonical autophagy in a SopF-sensitive fashion, in a process thus

124 termed V-ATPase-ATG16L1 induced LC3B lipidation (VAIL) (Fischer et al., 2020).

125 Integrating these diverse observations, we hypothesised that V-ATPase may represent a

126 universal regulator of non-canonical autophagy.

128 In this study, we investigate the role of V-ATPase in CASM, identifying a requirement for 129 enhanced V0-V1 association and direct ATG16L1 engagement across a wide range of non130 canonical autophagy processes. We also define the interrelationships between V-ATPase, 131 NADPH Oxidase and ROS during LAP, providing new mechanistic insight into this specific process. Finally, we explore the roles of V-ATPase and non-canonical autophagy during

133 Salmonella infection, concluding that CASM represents a host pathogen response, evaded by 134 the SopF effector.

\section{Results}

\section{$\mathrm{V}$-ATPase is recruited to phagosomes prior to $\mathrm{LC} 3$ lipidation}

140 During LAP, the fusion of phagosomes with late endosomes/lysosomes, and their associated acidification via V-ATPase, is assumed to occur after the recruitment of LC3, as a terminal

142 step (Martinez et al., 2015). However, inhibition of V-ATPase with Bafilomycin A1 (BafA1)

143 blocks LC3 recruitment during LAP, and other non-canonical autophagy processes, 
suggesting V-ATPase may play an additional, upstream role (Florey et al., 2015). To investigate this notion, we revisited the dynamics of LAP using live imaging.

First, fluorescently tagged markers of late endosomes/lysosomes were monitored, in relation to GFP-hLC3A, during phagocytosis of opsonised zymosan particles (LAP). Strikingly, we observed early recruitment of the late endosome marker, RFP-Rab7, to newly formed phagosomes, occurring prior to GFP-hLC3A recruitment (Fig. 1 a; and Video 1). Similarly, the lysosome marker, LAMP1-RFP, is also recruited to phagosomes before GFP-hLC3A (Fig. 1 b; and Video 2). By measuring the duration between onset of markers, we confirmed that phagosomes mature through a Rab7 - LAMP1 - LC3 sequence (Fig. 1 c). The same maturation profile was also observed during entosis, another macro-scale engulfment process that utilises non-canonical autophagy (Fig. S1). Together, these data imply that late endosomes/lysosomes fuse with the phagosome prior to the initiation of non-canonical autophagy signalling.

We next sought to visualise V-ATPase directly. This large, multi-subunit protein complex can be challenging to monitor in live cells, as fluorescent tags may interfere with function. Therefore, to assess V-ATPase and LC3 recruitment to phagosomes, immunofluorescent staining was performed at specific timepoints on fixed cells. At 20 minutes post zymosan incubation, the majority of phagosomes were positive for both the V1A subunit of V-ATPase and GFP-hLC3A (Fig. $1 \mathrm{~d}$ and e). Importantly, significantly more V1A positive, GFP-hLC3A negative phagosomes were detected than V1A negative, GFP-hLC3A positive (Fig. 1d and e), suggesting that V1A is likely to be recruited first. We can exclude the possibility that GFP-hLC3A has transiently translocated before fixation, as GFP-hLC3A clearly persisted on phagosomes for longer than 20-minutes in live imaging studies. Therefore, our data indicate that, like Rab7 and LAMP1, V-ATPase is present on phagosomes prior to LC3. This is consistent with previous work indicating that V-ATPase recruits to phagosomes at an early stage of their maturation (Sun-Wada et al., 2009).

Together, these data indicate that components of the late endosome/lysosome network, including V-ATPase, are recruited to phagosomes before the onset of LC3 lipidation during LAP. These data question the view that V-ATPase performs an exclusively terminal role, and are consistent with a possible upstream, regulatory function. 
NADPH oxidase and V-ATPase activities are both required for LAP

179 A major molecular mechanism implicated in LAP involves NADPH oxidase-mediated ROS

180 generation (Martinez et al., 2015); however, the specific mechanistic role of ROS remains

181 unclear. We next considered the possibility of a relationship between NADPH oxidase and

182 V-ATPase during LAP. Using the well characterised pharmacological inhibitors

183 Diphenyleneiodonium (DPI) and Bafilomycin A1 (BafA1), to inhibit NADPH oxidase or V-

184 ATPase respectively, a reduction in GFP-hLC3A recruitment to phagosomes was detected with both (Fig. 2 a). Notably, analysis of fluorescent intensity indicates that BafA1 yields the more pronounced inhibition (Fig. 2 b). These data confirm that both NADPH oxidase and VATPase are required for LAP.

Next, the effect of each inhibitor on phagosomal ROS generation was analysed, using

190 Nitroblue Tetrazolium (NBT) (Fig. 2 c) and luminol based assays (Fig. 2 d). As shown in Fig.

$1912 \mathrm{c}, \mathrm{O}_{2}{ }^{-}$mediated insoluble formazan deposition, in zymosan containing phagosomes, is abolished by DPI, but not by BafA1 treatment. In agreement with this, DPI treatment blocks zymosan induced ROS production, while BafA1 has no effect (Fig. 2 d). Together, these data establish that while NADPH oxidase is required for ROS generation in phagosomes, VATPase activity is not. Moreover, these results show that V-ATPase inhibition blocks LAP, even in the presence of a ROS burst. As such, we can deduce that NADPH oxidase-mediated ROS generation is not sufficient to induce LAP, and that V-ATPase acts either downstream, or in parallel, to promote LC3 lipidation to the phagosome membrane.

\section{ROS modulates phagosome acidification to drive LAP}

To test whether NADPH oxidase might function directly upstream of V-ATPase during LAP, the relationship between ROS and phagosome acidification was assessed, using Lysotracker and live cell confocal microscopy, in the presence or absence of DPI. Strikingly, inhibition of NADPH oxidase significantly increases Lysotracker intensity, signifying increased acidification of zymosan containing phagosomes by V-ATPase (Fig. 3 a). These data agree with previous studies (Mantegazza et al., 2008; Savina et al., 2006), which proposed that production of phagosomal ROS can constrain acidification, by increasing membrane permeability or through the consumption of protons $(\mathrm{H}+)$ (Westman and Grinstein, 2020). According to this model, in the absence of ROS, more protons can accumulate, and thus the phagosome is more readily acidified. 
212 To explore this further, we utilised chloroquine (CQ), a weak-base amine, which becomes

213 protonated and accumulates in acidifying compartments, thereby 'trapping' $\mathrm{H}+$ ions. We

214 reasoned that CQ could artificially sequester $\mathrm{H}+$, in DPI-treated cells, providing an

215 alternative way to limit protons, in the absence of ROS. In line with our predictions, the

216 addition of CQ countered the DPI-mediated increase in Lysotracker staining (Fig. $3 \mathrm{~b}$ and c).

217 Importantly, CQ treatment also rescued GFP-hLC3A recruitment in the presence of DPI,

218 connecting ROS generation and proton concentration directly to LAP (Fig. 3 b and d).

219 Together, these data establish a relationship whereby high phagosomal ROS levels raise $\mathrm{pH}$

220 and drive LAP.

222 To interrogate this relationship in the absence of pharmacological manipulation, bone marrow-derived murine macrophage (BMDM) and dendritic cells (BMDC) were compared.

224 Previous studies established that mouse BMDC phagosomes generate higher ROS levels, and 225 have a more neutral pH, than BMDM phagosomes (Mantegazza et al., 2008; Savina et al., 2006). Using the luminol assay to measure ROS, and Lysotracker staining to monitor acidification, we confirmed these observations (Fig. 3 e-g). Strikingly, we also found that BMDCs support higher levels of LAP than BMDMs (Fig. $3 \mathrm{~h}$ and i), consistent with our model.

Thus, using both pharmacological and cell type-based manipulations, our data indicate that increased LAP is associated with higher ROS production and $\mathrm{pH}$. These findings provide new insight into the molecular mechanisms underlying LAP and uncover a clear link between NADPH oxidase and V-ATPase activities.

\section{ROS modulates V-ATPase subunit recruitment}

237 We next sought to understand how ROS alters V-ATPase function, and how this results in the

238 lipidation of LC3 to phagosomes. While modulation of phagosome $\mathrm{pH}$ does occur with ROS, our data do not support this as a direct mechanism. Firstly, while DPI and BafA1 both inhibit LAP, they yield different effects on phagosomal pH (Fig. S2). Secondly, while BafA1 blocks LAP, monensin and many other drugs that raise lysosomal $\mathrm{pH}$, instead activate non-canonical autophagy (Jacquin et al., 2017). We therefore considered whether other, pump-independent features of V-ATPase might connect ROS and LAP, focussing first on V-ATPase subunit

244 localisation and recruitment. 
During LAP, we observe robust recruitment of ATP6V1A, a subunit of the V-ATPase V1 sector, to zymosan containing phagosomes (Fig. 4 a). Notably, both DPI and BafA1 treatment reduce this ATP6V1A signal intensity (Fig. $4 \mathrm{a}$ and b). This result may at first appear somewhat counter intuitive, given that DPI increases the acidification of phagosomes (Fig. 3 a). However, we speculate that in the absence of $\mathrm{H}+$ consumption by ROS, the phagosome requires less V-ATPase activity to support acidification, and therefore recruits less ATP6V1A. In support of this interpretation, artificial sequestration of $\mathrm{H}+$ by $\mathrm{CQ}$ reverses the DPI induced reduction in ATP6V1A staining (Fig. 4 c). To extend these observations, the recruitment of ATP6V1A to phagosomes was also compared in BMDCs and BMDMs. Consistent with the above data, BMDC phagosomes exhibit greater ATP6V1A recruitment than BMDMs (Fig. $4 \mathrm{~d}$ and e).

Taken together, these findings indicate that LAP activation correlates with V-ATPase V1 sector recruitment to the phagosome, which can be regulated by NADPH oxidase activity.

\section{Increased V0-V1 association drives non-canonical autophagy}

V-ATPase activity can be regulated by the association of the cytosolic V1 sector with the membrane bound $\mathrm{V} 0$ sector to form a functional holoenzyme, a requisite for pump activity (Collins and Forgac, 2020). To address whether regulated V0-V1 association might play a role in non-canonical autophagy, two structurally distinct V-ATPase inhibitors, BafA1 and Saliphenylhalamide (SaliP), were exploited. These inhibitors bind to distinct sites on VATPase, both blocking pump activity (Xie et al., 2004), and raising lysosomal pH. Importantly, however, while BafA1 acts to dissociate V0-V1, SaliP instead drives their association through a covalent adduct formation (Fig. 5 a) (Kissing et al., 2015; Xie et al., 2004). As such, we can use these inhibitors to differentially manipulate V0-V1 association.

Treatment of wild type MCF10A cells with BafA1 or SaliP leads to an increase in GFPhLC3A lipidation (Fig. 5 b), which would typically be attributed to the inhibition of autophagic flux (Yamamoto et al., 1998). Consistent with this, in ATG13KO cells, which are deficient in canonical autophagy, BafA1 yields no such increase in GFP-hLC3A lipidation. Strikingly, however, SaliP still induces robust GFP-hLC3A lipidation, co-localised with LAMP1, in the autophagosome-deficient ATG13 KO cells (Fig. 5 b and c). These data indicate that SaliP induces GFP-hLC3A lipidation through CASM, and that forced V0-V1 association may be sufficient to trigger non-canonical autophagy. 
281 To investigate this notion further, V0-V1 association was monitored by membrane

282 fractionation in response to known inducers of CASM. Strikingly, both monensin and CQ,

283 two distinct pharmacological agents which disrupt lysosomal ion balance to activate non-

284 canonical autophagy, promote increased V0-V1 association (Fig. $5 \mathrm{~d}$ and e). Furthermore, a

285 TRPML1 agonist (C8), which also drives CASM (Goodwin et al., 2021), similarly enhances

286 V0-V1 binding (Fig. 5 f). Notably however, activation of canonical autophagy, via mTOR

287 inhibition (AZD8055), does not increase V0-V1 engagement, suggesting this mechanism is specific to the non-canonical pathway.

Taken together, these data indicate that enhanced V0-V1 engagement is a conserved feature of non-canonical, but not canonical, autophagy processes. Based on the use of SaliP, forced association of V0-V1 is sufficient for CASM activation, and independent of V-ATPase pump activity. We conclude that non-canonical autophagy depends instead upon physical subunit engagement, likely associated with structural changes within the V-ATPase complex itself.

\section{V0-V1 association drives V-ATPase - ATG16L1 interaction}

A direct interaction between V-ATPase and ATG16L1 was recently identified in the context of xenophagy (Xu et al., 2019). Intriguingly, this interaction involves the WD40 CTD of ATG16L1, a domain essential for CASM (Fletcher et al., 2018). We thus hypothesized that V-ATPase, and specifically V0-V1 engagement, may drive CASM via a comparable direct recruitment of ATG16L1. To test this, co-immunoprecipitation experiments were performed in cells expressing either FLAG-tagged WT ATG16L1, or a WD40 CTD point mutant (K490A), which is specifically deficient in non-canonical autophagy (Durgan et al., 2021; Fletcher et al., 2018). Under resting conditions, ATG16L1 does not interact with V-ATPase. However, during LAP, we observe a robust interaction between WT ATG16L1 and the VATPase ATP6V1A subunit (Fig. 6 a and e). Importantly, this interaction is completely abolished by the K490A mutation, consistent with the molecular dependencies of noncanonical autophagy, while both wild type and K490A ATG16L1 are able to pull down ATG5. This K490-dependent interaction between ATG16L1 and ATP6V1A is similarly induced by diverse non-canonical autophagy activators including the STING ligand DMXAA

311 (Fig. 6 b and e), TRPML1 activation with C8 (Fig. $6 \mathrm{c}$ and f), monensin (Fig. S3) and

312 influenza A virus infection (data in submission). Strikingly, however, activation of canonical 313 autophagy via mTOR inhibition (PP242), does not induce ATG16L1 - V-ATPase interaction 
314 (Fig. $6 \mathrm{~d}$ and e), suggesting again that this mechanism is associated specifically with CASM.

315 Finally, we find that treatment of cells with SaliP can also promote V-ATPase-ATG16L1

316 binding, dependent on K490A, indicating that enhanced V0-V1 association is sufficient to

317 drive this interaction (Fig. $6 \mathrm{~g}$ ).

319 Together, these data reveal that V-ATPase directly recruits ATG16L1 during diverse non-

320 canonical autophagy processes to drive CASM. We speculate that V0-V1 association permits

321 this interaction, perhaps via a conformational change, and that it requires that the K490

322 containing pocket, on the top face of the ATG16L1 C terminal WD40 $\beta$ barrel. We propose

323 that during CASM, the V-ATPase complex may play an analogous role to WIPI2 in

324 canonical autophagy, recruiting ATG16L1 to the appropriate membrane, to specify the site of

325 ATG8 conjugation.

326

\section{SopF blocks LAP and non-canonical autophagy}

328 SopF is a Salmonella effector protein that blocks the interaction between V-ATPase and 329 ATG16L1 during xenophagy, by ribosylation of Gln124 in the ATP6V0C subunit (Xu et al.,

330 2019). We reasoned that if V-ATPase drives LAP through recruitment of ATG16L1, then this 331 would also be sensitive to SopF. Strikingly, transient overexpression of mCherry-SopF in

332 RAW264.7 cells completely inhibits GFP-hLC3A lipidation during phagocytosis of zymosan

333 (Fig. 7 a and b), without affecting ROS production in phagosomes, as determined by NBT

334 test (Fig. 7 c). These data are consistent with a model in which ROS acts upstream of V-

335 ATPase to modulate ATG16L1 recruitment. Interestingly, stable expression of SopF in

336 MCF10A cells does not interfere with V0-V1 association, as promoted by monensin or SaliP

337 (Fig. 7 d), suggesting it specifically blocks downstream ATG16L1 engagement. Notably,

338 SopF also appears to inhibit non-canonical LC3 lipidation in the context of STING or

339 TRPML1 activation and influenza A virus infection (Fischer et al., 2020; Goodwin et al.,

340 2021; Ulferts et al., 2020). Taken together, our data, alongside these recent studies, support

341 the conclusion that V-ATPase is a universal regulator of CASM, which functions to recruit

342 ATG16L1, in a SopF-sensitive fashion.

344 Non-canonical autophagy contributes to the Salmonella host response

345 Finally, we considered the mechanistic links between V-ATPase, ATG16L1, CASM and

346 SopF in the functional context of host pathogen responses. Salmonella SopF provides a 
mechanism to evade host LC3 lipidation, and this has been elegantly studied in the context of xenophagy (Xu et al., 2019). Here, we investigated the hypothesis that SopF may also suppress CASM as a parallel host response. To explore a possible role for non-canonical autophagy during Salmonella infection, HCT116 cells, expressing wild type or K490A ATG16L1, were infected with either wild type or $\Delta s o p F$ Salmonella. In agreement with Xu et $a l$, we found GFP-rLC3B recruitment to Salmonella is increased using the $\Delta s o p F$ strain in wild type cells (Fig. 8 a-c). Importantly, we also found that in non-canonical autophagy deficient ATG16L1 K490A cells, GFP-rLC3B recruitment to either strain is dramatically reduced (Fig. 8 a-c). Considering the K490A mutation has no effect on canonical autophagy, or autophagosome formation (Fletcher et al., 2018; Rai et al., 2019) (Fig. 6 e), these data strongly suggest that Salmonella infection also induces a non-canonical autophagy response, which is disrupted by SopF targeting of the V-ATPase-ATG16L1 axis. Together, these findings indicate that Salmonella infection triggers CASM as a defensive host response, and that SopF has evolved as a mechanism of evasion.

\section{Discussion}

Non-canonical autophagy, or CASM, has emerged as a pathway with vital functions in a wide range of biological processes, including immunity, vision and cancer. A number of molecular players have been implicated in regulation of this pathway, including the core ATG8 conjugation machinery, NADPH oxidase, Rubicon and V-ATPase. However, exactly how these components work together, and whether they are context-specific or common to all forms of CASM, has remained unclear. In this study, we focussed on the involvement of the V-ATPase complex, its interplay with ATG16L1 during non-canonical autophagy processes, and its specific connection to NADPH oxidase/ROS during LAP.

V-ATPase was first implicated in non-canonical autophagy through pharmacological studies that demonstrated opposing effects of BafA1 and CQ on CASM, during LAP and entosis (Florey et al., 2015). BafA1 and CQ are both lysosomal inhibitors, but with different mechanisms of action. BafA1 functions as a V-ATPase inhibitor, and blocks CASM, while CQ, which acts instead to quench protons in the lysosome, induces CASM, in a BafA1 sensitive fashion. These findings indicated that CASM responds to the lumenal ionic balance 
in lysosomes, and that V-ATPase is essential for the lipidation of ATG8s to single membranes during non-canonical autophagy.

This notion was somewhat unexpected, as V-ATPase is typically thought of as a downstream player during autophagy-related processes, driving terminal lysosomal degradation. However, an upstream, regulatory role for V-ATPase was further reinforced by the finding that, like CQ, a wide range of other ionophores and lysosmotropic agents, including monensin, nigericin, CCCP and clinically used drugs such as lidocaine and amiodarone, similarly induce CASM, in a BafA1 sensitive manner (Jacquin et al., 2017). Furthermore, a comparable pharmacological profile has also been observed during non-canonical autophagy following STING activation, through a process thus termed VAIL (Fischer et al., 2020), and following TRPML1 activation (Goodwin et al., 2021).

Despite the growing evidence that V-ATPase is required to induce non-canonical autophagy, exactly how this complex might sense and respond to different stimuli, and activate CASM, remained unknown. In this study, we have defined a key regulatory mechanism (Fig. 8). Specifically, we find that neutralization of endolysosomal compartments leads to an increased association of the V-ATPase V0-V1 subunits, which in turn facilitates direct ATG16L1 recruitment, through a K490-dependent interaction with the ATG16L1 C terminal WD domain. This mechanism operates in all tested examples of non-canonical autophagy, suggesting this represents a universal regulatory mechanism for the pathway.

Activation of non-canonical autophagy may thus represent a cellular response to stressed or perturbed endolysosomal compartments, that transduce this signal via increased V0-V1 association. It seems likely that these stresses, including lumenal $\mathrm{pH}$ change or ionic imbalance, may involve different mechanisms depending on the specific stimulus. For example, pharmacological ionophores and lysosomotropic drugs directly neutralize endolysosomal compartments, which then increase V0-V1 association in an attempt to reacidify; the ability of V-ATPase to sense and respond to altered lumenal $\mathrm{pH}$ in this way has been previously proposed (Marshansky, 2007). In a similar way, the influenza A virus M2 protein, which acts as a $\mathrm{H}+$ proton ionophore, neutralizes intracellular vesicles to activate CASM (Fletcher et al., 2018; Ulferts et al., 2020). 
412 During the activation of LAP, our data indicate that phagosome $\mathrm{pH}$ is neutralized by the high

413 levels of ROS generated by NADPH oxidase. The ROS consume protons, thereby raising $\mathrm{pH}$

414 and driving increased phagosomal V1 levels in response. By linking together NADPH

415 Oxidase, ROS and V-ATPase, these findings reveal direct connections between some of the

416 key players in LAP, thus building towards a unifying mechanism. We note that phagosomal

417 ROS have also been linked to the inhibition of ATG4 deconjugation activity during LAP

418 (Ligeon et al., 2021), which may perhaps be related more to prolonging ATG8 lipidation at

419 phagosomes than to its induction.

420

421 Further work will be required to assess the triggers for enhanced V0-V1 association in other

422 contexts. In the case of entosis, which involves degradation of an entire internalised cell, it is

423 tempting to speculate that the level of V-ATPase activity required to acidify such a large

424 compartment may necessitate enhanced V0-V1 association. During VAIL, the trafficking of

425 STING to endolysosomes, or TBK1 phosphorylation, may be involved. However, overall, it

426 appears likely that while multiple routes exist to trigger CASM at endolysosomal

427 compartments, these all converge at V-ATPase, and specifically upon enhanced V0-V1

428 association, as a common step in non-canonical autophagy.

429

430 Importantly, a direct interaction between V-ATPase and the WD40 C terminal domain (CTD) 431 of ATG16L1 was recently identified during Salmonella xenophagy (Xu et al., 2019). In this

432 study, we report that this interaction is also induced across a range of non-canonical

433 autophagy processes, including LAP, activation of TRPML1 or STING, and drug-induced

434 CASM (monensin), but importantly, not during canonical autophagy. We show that

435 ATG16L1 binding occurs upon enhanced V0-V1 association, in a pump-independent manner,

436 and indeed that increased V0-V1 engagement, triggered by SaliP, is sufficient to recruit

437 ATG16L1. Furthermore, we map the interaction to a specific residue in the ATG16L1 WD40

438 CTD (K490). Together, these data indicate that the interaction between V-ATPase and

439 ATG16L1 is universally required for non-canonical autophagy. We speculate that V-ATPase

440 may perform a similar function to WIPI2 in canonical autophagy, specifying the site of

441 ATG16L1 recruitment to direct ATG8/LC3 lipidation during CASM.

443 The Salmonella effector SopF ribosylates Gln124 of ATP6V0c in the V-ATPase, inhibiting

444 its interaction with ATG16L1 during xenophagy (Xu et al., 2019), and also during STING

445 activation (Fischer et al., 2020), Influenza virus A infection (Ulferts et al., 2020) and a range 
of other CASM processes, as shown here. Further to this, our data show that while SopF blocks CASM, it does not block increased V0-V1 association. These findings suggest a specific sequence of events during CASM (Fig. 8), and the existence of a SopF-sensitive conformational change, that occurs during increased V-ATPase activity and V0-V1 association, to engage ATG16L1. Using cryo-electron microscopy, recent advances have been made in determining the complete mammalian structure of V-ATPase (Wang et al., 2020a; Wang et al., 2020b), where different holoenzyme states have been identified. In the future it may be possible to resolve specific structural features in SopF-modified versus unmodified complexes, that are required to support ATG16L1 WD40 CTD binding.

V-ATPase activity is well known to be regulated along the endocytic pathway, with increased assembly and lower pH as it progresses towards lysosomes (Lafourcade et al., 2008). However, the molecular mechanisms regulating V0-V1 association in mammalian cells remain poorly understood. Glucose and amino acid availability can modulate the assembly and association of the V-ATPase complex (McGuire and Forgac, 2018; Stransky and Forgac, 2015), in a PI3-kinase dependent manner, and lipid composition is proposed to play a regulatory role, with $\mathrm{PI}(3,5) \mathrm{P} 2$ stabilising the V0-V1 association (Li et al., 2014). Changes in V0-V1 levels have also been reported in different differentiation states of murine dendritic cells, with more mature cells displaying increased V0-V1 association (Liberman et al., 2014); our data now reveal an increased V1A recruitment to phagosomes in bone marrow-derived dendritic cells compared to macrophage. Thus, it seems likely that differences in noncanonical autophagy activation may be observed between different cell types and species, dependent on basal differences in their regulation of V-ATPase.

While non-canonical autophagy regulates a wide range of fundamental biological processes (Heckmann et al., 2017), the underlying molecular and cellular functions of CASM are still not fully understood. During LAP, ATG8 recruitment has been proposed to modulate phagosome maturation and content degradation. However, while some studies suggest a role for ATG8s in promoting phagosome maturation and lysosome fusion (Gluschko et al., 2018; Henault et al., 2012; Martinez et al., 2015), others suggest instead that ATG8 recruitment delays maturation to stabilise phagosomes (Romao et al., 2013). In another study, no gross defect in phagosome maturation was detected upon inhibition of ATG8 lipidation (Cemma et al., 2016), and, BafA1, which inhibits LAP, does not impair the recruitment of LAMP1 to phagosomes (Kissing et al., 2015). Considering our new data, which show that ATG8/LC3 is 
480

481

482

483

484

485

486

487

488

489

490

491

492

493

494

495

496

497

498

499

500

501

502

503

504

505

506

507

508

509

510

511

512

recruited to phagosomes after the acquisition of late endosome/lysosome markers, it seems possible that LAP may act to fine tune, rather than initiate, phagosome maturation. For instance, perhaps ATG8/LC3s recruit interacting proteins to modulate the extent of lysosome fusion and/or signalling from phagosomes. Further work will be required to resolve these debates in the literature and to elucidate possible cell type or context specific differences.

Non-canonical autophagy and the ATG16L1-V-ATPase axis facilitate a key innate immune response to pathogen infection. As such, pathogens have evolved evasion strategies, which further underscore the functional importance of CASM. The intracellular pathogen, Legionella pneumophila, expresses an effector protein, RavZ, which deconjugates ATG8 from both PE and PS (Durgan et al., 2021), and blocks LAP (Martinez et al., 2015). Cell wall melanin from the fungal pathogen, Aspergillus fumigatus, inhibits LAP by interfering with NADPH oxidase and ROS production (Akoumianaki et al., 2016; Kyrmizi et al., 2018). As noted above, the Salmonella effector protein, SopF, directly targets the ATG16L1-V-ATPase interaction to permit bacterial growth (Xu et al., 2019); to date, this effect has been attributed solely to xenophagy inhibition. Here, using genetic models to specifically inhibit noncanonical autophagy, we build on these findings to show that SopF also inhibits CASM, and suggest that the ATG8 response to Salmonella is, in part, due to non-canonical autophagy. These data are consistent with other studies implicating LAP in Salmonella infection (Masud et al., 2019). In the future it would be of interest to explore the impacts of other pathogen effectors on CASM, such as VopQ from Vibrio parahaemolyticus and SidK from Legionella, both of which target V-ATPase.

In conclusion, we have defined a key molecular mechanism unifying the various forms of non-canonical autophagy, whereby enhanced V0-V1 association recruits ATG16L1 to VATPase positive, endolysosomal compartments to drive CASM. This mechanism explains the unusual pharmacological profile of CASM processes, and provides new molecular insight into how NADPH oxidase and ROS activate LAP. These findings also build on the recent elucidation of V-ATPase-ATG16L1 binding during Salmonella induced xenophagy, indicating that this interaction also occurs during non-canonical autophagy, and indeed that V-ATPase specifies the site of single membrane ATG8 lipidation during CASM. Finally, our data implicate CASM as a parallel pathogen response during Salmonella infection, blocked by the effector protein SopF. 


\section{Acknowledgements}

514

515 We thank Nick Ktistakis, Simon Cook and Phill Hawkins for helpful discussions and critical

516 review of the manuscript, and the BI Imaging facility for all their support. This work was

517 supported by grants from the BBSRC, BB/P013384/1 (BBS/E/B/000C0432 and

518 BBS/E/B/000C0434), BB/R019258/1 and Cancer Research UK Career Development award

519 C47718/A16337. J.H.B. holds the Pitblado Chair in Cell Biology. Work in the lab of J.H.B.

520 was supported by an operating grant from the Canadian Institutes of Health Research

521 (FDN\#154329).

522

523

524

525 Contributions

526

527 K.M.H and E.J. designed and carried out experiments. T.L. and J.H.B. carried out and 528 analysed Salmonella experiments. J.M.G provided reagents and insights and performed

529 preliminary experiments. J.D. designed experiments and wrote the manuscript. O.F. designed

530 and carried out experiments and wrote the manuscript. 


\section{Antibodies}

534 Antibodies used were rabbit polyclonal anti-ATG16L1 (8090, Cell Signalling, WB 1:1000),

535 rabbit polyclonal anti-ATG5 (2630, Cell Signalling, WB 1:1000), rabbit polyclonal anti-

536 LC3A/B (4108, Cell Signalling, WB 1:1000, IF 1:100), rabbit monoclonal anti-ATP6V1A

537 (ab199326, Abcam, WB 1:2000, IF 1:250), mouse monoclonal anti-ATP6V0d1 (ab56441,

538 Abcam, WB 1:1000), mouse monoclonal anti-LAMP1 (555798, BD Biosciences, IF 1:100),

539 mouse monoclonal anti-mCherry (ab125096, Abcam, WB 1:1000), mouse monoclonal anti-

540 GAPDH (ab8245, Abcam, WB 1:1000), Alexa Fluor 488 polyclonal goat anti-rabbit IgG (A-

541 11034, ThermoFisher, IF 1:500), Alexa Fluor 568 polyclonal goat anti-mouse IgG (A-11004,

542 ThermoFisher, IF 1:500), Alexa Fluor 568 polyclonal goat anti-rabbit IgG (A-11011,

543 ThermoFisher, IF 1:500), HRP-conjugated anti-rabbit IgG (7074, Cell Signalling, WB

544 1:2000), HRP-conjugated anti-mouse IgG (7076, Cell Signalling, WB 1:2000).

545

546 Reagents

547 Reagents and chemicals used were BafA1 (1334, Tocris, 100 nM), PP242 (4257, Tocris, 1

$548 \mu \mathrm{M})$, AZD8055 (S1555, Selleckchem, $1 \mu \mathrm{M})$, Monensin (M5273, Sigma, $100 \mu \mathrm{M})$, DPI

549 (D2926, Sigma, 5 MM), human serum (H2918, Sigma), DMXAA (D5817, Sigma, 50 mg/ml),

550 Zymosan (Z4250, Sigma), NBT (N6876, Sigma), murine IFN $\gamma$ (315-05, Peprotech), DAPI

551 (D9542, Sigma), IN-1 (17392, Caymen, $1 \mu \mathrm{M}$ ), LysoTracker Red DND-99 (L7528,

552 ThermoFisher), LysoTracker Deep Red (L12492, ThermoFisher), anti-FLAG M2 magnetic

553 beads (M8823, Sigma). Saliphenyhalamide (SaliP, $2.5 \mu \mathrm{M})$ and TRPML1 agonist C8 (2 $\mu \mathrm{M})$

554 were provided by Casma Therapeutics, USA.

555

\section{Plasmid constructs}

557 pmCherry-SopF was generated previously (Lau et al., 2019) and kindly provided by Dr Leigh

558 Knodler. mCherry-SopF was cloned into pQCXIP using AgeI/BamHI restriction sites.

559 mRFP-Rab7 was a gift from Ari Helenius (Addgene plasmid \# 14436). pBabe-Puro-RFP-

560 LAMP1 was kindly provided by Dr Michael Overholtzer.

\section{Cell culture}


WT or $A T G 13^{-/}$MCF10A cells (human breast epithelial), expressing GFP-LC3A (human), were prepared as described previously (Jacquin et al., 2017) and cultured in DMEM/F12 (Gibco, 11320074) containing 5\% horse serum (ThermoFisher, 16050-122), EGF (20 ng/ml;

566 Peprotech AF-100-15), hydrocortisone (0.5 mg/ml; Sigma, H0888), cholera toxin (100 ng/ml;

567 Sigma, C8052), insulin (10 $\mu \mathrm{g} / \mathrm{ml}$; Sigma, I9278), and penicillin/streptomycin (100 U/ml;

568 Gibco $15140-122$ ) at $37^{\circ} \mathrm{C}, 5 \% \mathrm{CO}_{2}$.

569

HCT116 cells (human colorectal epithelial) were maintained using DMEM (Gibco, 41966029) supplemented with 10\% FBS (Sigma, F9665) and penicillin/streptomycin (100 U/ml, $100 \mu \mathrm{g} / \mathrm{ml}$; Gibco $15140-122)$ at $37^{\circ} \mathrm{C}, 5 \% \mathrm{CO}_{2}$. A panel of HCT116 lines expressing GFPLC3B (rat) and different ATG16L1 constructs, were derived from ATG16L $1^{-/}$cells, reconstituted with pBabe-Puro FLAG-S-ATG16L1 (wild type or K490A), as described previously (Fletcher et al., 2018).

RAW264.7 cells were obtained from ATCC and maintained in DMEM (Gibco, 41966-029) supplemented with 10\% FBS (Sigma, F9665) and penicillin/streptomycin (100 U/ml, 100 $\mu \mathrm{g} / \mathrm{ml}$; Gibco $15140-122)$ at $37^{\circ} \mathrm{C}, 5 \% \mathrm{CO}_{2}$. ATG16L1/- were generated and reconstituted with pBabe-Puro FLAG-S-ATG16L1 (wild type or K490A), and GFP-LC3A (human) as described previously (Durgan et al., 2021; Lystad et al., 2019).

582

\section{Retrovirus generation and infection}

584 For mCherry-SopF virus infection, MCF10A cells were seeded in a 6 well plate at $5 \times 10^{4}$ per well. The next day $1 \mathrm{ml}$ viral supernatant was added with $10 \mu \mathrm{g} / \mathrm{ml}$ polybrene for $24 \mathrm{~h}$ followed by a media change. Cells were then selected with puromycin $(2 \mu \mathrm{g} / \mathrm{ml})$.

\section{BMDC and BMDM isolation}

589 Bone marrow extracted from the hind legs of one C57/BL6 mouse was resuspended in $10 \mathrm{ml}$ complete RPMI 1640 media (Sigma, R8578) (10\% FBS, 1\% P/S, 50 M 2-mercaptoethanol) then centrifuged at $1500 \mathrm{rpm}$ for $5 \mathrm{~min}$. The bone marrow was then resuspended in $1 \mathrm{ml}$ of red blood cell lysis buffer $\left(168 \mathrm{mM} \mathrm{NH}_{4} \mathrm{Cl}, 100 \mu \mathrm{M} \mathrm{KHCO}_{3}, 10 \mathrm{mM} \mathrm{Na} 2 \mathrm{EDTA}(\mathrm{pH}\right.$ 8), in milliQ $\mathrm{H}_{2} \mathrm{O}$, final $\mathrm{pH} 7.3$ ) and agitated for 2 min prior to being resuspended in $10 \mathrm{ml}$ complete RPMI 1640 media and centrifuged at $1500 \mathrm{rpm}$ for $5 \mathrm{~min}$. For dendritic cell 
supplemented with 20 ng/ml GM-CSF (Peprotech \#315-03), 10 ng/ml IL-4 (Peprotech \#AF214-14) and $50 \mathrm{ng} / \mathrm{ml}$ Amphotericin B (Gibco \#15290018). For macrophage differentiation the bone marrow was resuspended in $25 \mathrm{ml}$ of complete RPMI 1640 media supplemented with $20 \mathrm{ng} / \mathrm{ml}$ M-CSF (Peprotech \#AF-315-02) and $50 \mathrm{ng} / \mathrm{ml}$ Amphotericin B. The cells were incubated for 3 days at $37^{\circ} \mathrm{C}, 5 \% \mathrm{CO}_{2}$, in low-adherence $90 \mathrm{~mm}$ sterilin petri dishes (101R20, ThermoFisher). On the third day media was removed and centrifuged at $1500 \mathrm{rpm}$ for $5 \mathrm{~min}$.

602 The non-adherent cells were then resuspended in fresh differentiation media and added back to the dishes containing the adherent cells for a further 3 days of differentiation. On the sixth day cells non-adherent cells in the media are removed and adherent cells are harvested by gently scraping into complete RPMI 1640 media. The cells were then centrifuged at 1500 rpm for 5 min and seeded in complete RPMI 1640 media without differentiation cytokines.

607

\section{Amaxa nucleofection}

609 Transient transfection was performed by electroporation using a Nucleofector II instrument

610 (Lonza, Wakersville, MD, USA) and Lonza nucleofection kit V (Lonza, VCA-1003)

611 following manufacturer's guidelines. Briefly, 2 x 106 RAW264.7 were electroporated with 2

$612 \mu \mathrm{g}$ mCherry-SopF or RFP-Rab7 using programme D-032.

613

\section{Fluorescent Live cell microscopy and analysis}

615 Prior to imaging, RAW264.7 cells were plated overnight on $35 \mathrm{~mm}$ glass-bottomed dishes

616 (MatTek) in the presence of $200 \mathrm{U} / \mathrm{ml}$ IFN $\gamma$. Opzonised zymosan (OPZ) particles were

617 generated by incubating Zymosan A from Saccharomyces (Z4250, Sigma) in human serum

618 (P2918, Sigma) for $30 \mathrm{~min}$ at $37^{\circ} \mathrm{C}$. The zymosan was then centrifuged at 5,000 rpm for 5

$619 \mathrm{~min}$, and then resuspended in PBS at $10 \mathrm{mg} / \mathrm{ml}$. The solution was passed through a 25-gauge

620 needle using a $1 \mathrm{ml}$ syringe several times to break up aggregates. Cells were mounted on a 621 spinning disk confocal microscope, comprising a Nikon Ti-E stand, Nikon 60x 1.45 NA oil 622 immersion lens, Yokogawa CSU-X scanhead, Andor iXon 897 EM-CCD camera and Andor 623 laser combiner. All imaging with live cells was performed within incubation chambers at $62437^{\circ} \mathrm{C}$ and $5 \% \mathrm{CO}_{2}$. For RFP-Rab7, LAMP1-RFP and GFP-LC3 imaging, z stacks were 625 acquired every $30 \mathrm{sec}$ following addition of OPZ. Image acquisition and analysis was 626 performed with Andor iQ3 (Andor Technology, UK) and ImageJ. 
For analysis of GFP-LC3 recruitment to phagosomes, IFN $\gamma$ treated RAW264.7 cells pretreated with inhibitors as indicated, followed by stimulation with $\mathrm{OPZ}$ for $25 \mathrm{~min}$ at $37^{\circ} \mathrm{C} . \mathrm{Z}$ stacks from multiple fields of view were acquired by spinning disk confocal microscopy as described above. Maximum GFP intensity from line profiles made over multiple individual phagosomes from 3 independent experiments, were measured using Image J software.

634 For scoring cells with GFP-LC3 positive phagosomes, those cells containing 1 or more phagosomes were counted and scored positive if they contain any GFP-LC3 labelled phagosomes. More than 150 cells were analysed over 3 experiments.

638 For lysotracker imaging, IFN $\gamma$ treated RAW264.7 cells were incubated with LysoTracker Red $(50 \mathrm{nM})$ for $15 \mathrm{~min}$ at $37^{\circ} \mathrm{C}$. Z stacks were acquired over time, or after 25 mins stimulation with OPZ, by spinning disk microscopy as described above. Mean fluorescent intensity of individual phagosomes was measured using Image J software. For primary cells, Lysotracker Deep Red (50 nM) was used and images acquired using a Zeiss LSM 780 confocal microscope (Carl Zeiss Ltd), using Zen software (Carl Zeiss Ltd).

645 For entosis, MCF10A cells were plated on glass bottomed 6-well plates (MatTek). The next 646 day, cells were maintained in an incubation chamber at $37^{\circ} \mathrm{C}$ and $5 \% \mathrm{CO}_{2}$, and cell-in-cell structures imaged by widefield timelapse microscopy. Fluorescent and DIC images were acquired every 8 min using a Flash 4.0 v2 sCMOS camera (Hamamatsu, Japan), coupled to a Nikon Ti-E inverted microscope, using a $20 \times 0.45$ NA objective. Image acquisition and analysis was performed with Elements software (Nikon, Japan).

\section{Fixed immunofluorescent confocal microscopy and analysis}

653 Cells were seeded in 12-well plates containing coverslips and incubated at $37^{\circ} \mathrm{C}, 5 \% \mathrm{CO}_{2}$ for

$65424 \mathrm{~h}$. Following treatments, cells were washed twice with ice cold PBS then incubated with $100 \%$ methanol at $-20^{\circ} \mathrm{C}$ for $10 \mathrm{~min}$. The cells were then washed twice with PBS and blocked with $0.5 \%$ BSA (Sigma, A7906) in PBS for $1 \mathrm{~h}$ at room temperature. The cells were incubated overnight at $4^{\circ} \mathrm{C}$ with the primary antibodies, then washed $\mathrm{x} 3$ in cold PBS.

658 Fluorescent secondary antibodies were used at a 1:500 dilution in PBS $+0.5 \%$ BSA and were 659 incubated with the cells for $1 \mathrm{~h}$ at room temperature. The cells were washed $\mathrm{x} 3$ in cold PBS 
661

662

663

664

665

666

667

668

669

670

671

672

673

674

675

676

677

678

679

680

681

682

683

684

685

686

687

688

689

690

691

692

microscope slides with ProLong Gold anti-fade reagent (Invitrogen, P36930). Image acquisition was made using a Zeiss LSM 780 confocal microscope (Carl Zeiss Ltd), using Zen software (Carl Zeiss Ltd).

For primary cells, maximal ATP6V1A intensity was quantified at the phagosome membrane and divided by the mean intensity within the cell perimeter using Image $\mathrm{J}$ software. For each condition 10 phagosomes within 4 images were quantified and repeated in 3 independent experiments. For RAW264.7 cells, maximum intensity from line profiles made over individual phagosomes were measured from 3 independent experiments using Image $\mathbf{J}$ software.

\section{Salmonella infection}

Infections were performed with wild-type and $\Delta s o p F$ Salmonella enterica serovar Typhimurium SL1344 which are kind gifts from Dr. Feng Shao. Late-log bacterial cultures were used for infection during experiments as outlined previously (D'Costa et al., 2015). Briefly, GFP-LC3 expressing HCT116 cells were seeded on $1 \mathrm{~cm}^{2}$ glass coverslips in 24-well tissue culture plates $48 \mathrm{~h}$ before use at a density of 100,000 cells/coverslip. Bacteria were grown for $16 \mathrm{~h}$ at $37^{\circ} \mathrm{C}$ with shaking and then sub-cultured (1:33) in LB without antibiotics for $3 \mathrm{~h}$. Post subculture, bacteria were pelleted at 10,000g for $2 \mathrm{~min}$, resuspended and diluted 1:100 in PBS, pH 7.2, with calcium and magnesium, and added to cells for $10 \mathrm{~min}$ at $37^{\circ} \mathrm{C}$. The cells were then washed 3 times with PBS with calcium and magnesium. Selection for intracellular bacteria was performed at $30 \mathrm{~min}$ post-infection using $100 \mu \mathrm{g} / \mathrm{ml}$ gentamicin. At $1 \mathrm{~h}$ post-infection, cells were fixed with $4 \%$ paraformaldehyde in $\mathrm{PBS}$ at $37^{\circ} \mathrm{C}$ for $10 \mathrm{~min}$.

\section{Nitroblue tetrazolium (NBT)/formazan assay}

RAW264.7 cells plated on cover slips were incubated with $0.2 \mathrm{mg} / \mathrm{ml} \mathrm{NBT}$ for $10 \mathrm{~min}$ at $37^{\circ} \mathrm{C}$. Where indicated, cells were also pre-incubated with inhibitors prior to addition of OPZ for $25 \mathrm{~min}$. Samples were then fixed by ice cold methanol and dark formazan deposits detected by DIC imaging on a Zeiss LSM 780 confocal microscope (Carl Zeiss Ltd), using Zen software (Carl Zeiss Ltd).

\section{Luminol assay}


693

694

695

696

697

698

699

700

701

702

703

704

705

706

707

708

709

710

711

712

713

714

715

716

717

718

719

720

721

722

723

724

725

RAW264.7 cells were seeded in 96-well white microwell plates (ThermFisher, 136101) at $1 \times 10^{5}$ cells/well and incubated at $37^{\circ} \mathrm{C}, 5 \% \mathrm{CO}_{2}$ for $24 \mathrm{~h}$. Growth media was removed and cells were washed with PBS. Any pre-treatments were incubated at $37^{\circ} \mathrm{C}$, and a final concentration of $0.32 \mathrm{U} / \mathrm{ml}$ of HRP (Sigma, P8375) and a final concentration of $125 \mathrm{nM}$ of luminol (Sigma, A8511) diluted in DPBS (Dulbecco's Phosphate Buffered Saline with Ca2+ and with Mg2+, Sigma, D8662), supplemented with 45\% glucose solution (Sigma, G8769) and $7.5 \%$ sodium bicarbonate solution (Sigma, S8761), were added to the cells and incubated for the final 3 minutes of the pre-treatment incubation at $37^{\circ} \mathrm{C}$. Stimuli were then added in triplicate and measurements were acquired immediately in the MicroLumatPlus LB 96V (Berthold technologies) at $37^{\circ} \mathrm{C}$.

\section{Membrane fractionation}

MCF10A cells were seeded on a $15 \mathrm{~cm}$ dish and cultured for $48 \mathrm{~h}$. Cells were stimulated in suspension as indicated for $1 \mathrm{~h}$. Input, cytosol and membrane fractions were isolated using the Mem-Per Plus Membrane Protein Extraction Kit (89842, Thermofisher) following product guidelines. Protein concentration was measured by BCA assay and equal amounts loaded onto polyacrylamide gels for SDS-PAGE analysis.

\section{Immunoprecipitation}

One confluent $15 \mathrm{~cm}$ plate of HCT116 and RAW 264.7 cells was used per condition.

Following stimulation, cells were washed twice with ice-cold PBS then lysed in $700 \mu$ of lysis buffer (50 mM Tris-HCl (pH 7.5-7.6), $150 \mathrm{mM} \mathrm{NaCl,} 2$ mM EDTA, 0.8\% C12E9, and protease/phosphatase inhibitors) per plate, on ice, and incubated for $20 \mathrm{~min}$ at $4^{\circ} \mathrm{C}$. Lysates were then centrifuged at $13,500 \mathrm{rpm}$ for $10 \mathrm{~min}$ at $4^{\circ} \mathrm{C}$ and then rotated with $10 \mu \mathrm{l}$ of preequilibrated protein A beads per sample (Pierce Protein A Magnetic Beads, ThermoFisher $88845, \mathrm{RA} 228548$ ) for $1 \mathrm{~h}$ at $4^{\circ} \mathrm{C}$. Following separation by magnet, lysates were then rotated with $25 \mu$ of pre-equilibrated anti-FLAG® M2 Magnetic Beads per sample (Millipore, $\mathrm{M} 8823)$ for $2 \mathrm{~h}$ at $4^{\circ} \mathrm{C}$. The lysates were washes five times in adjusted lysis buffer $(50 \mathrm{mM}$ Tris-HCl (pH 7.5-7.6), $150 \mathrm{mM} \mathrm{NaCl}, 2$ mM EDTA, 0.1\% C12E9, and 1\% Triton X-100) by rotating for $10 \mathrm{~min}$ at $4^{\circ} \mathrm{C}$. Samples were eluted by boiling at $95^{\circ} \mathrm{C}$ in $25 \mu 12 x$ SDS-buffer for $10 \mathrm{~min}$.

\section{Western blotting}


726 Cells were scraped into ice-cold RIPA buffer (150 mM NaCl, $50 \mathrm{mM}$ Tris- $\mathrm{HCl}, \mathrm{pH} 7.4$,

7271 mM EDTA, 1\% Triton X-100 (Sigma, T8787), 0.1\% SDS (Sigma, L3771), 0.5\% sodium

728 deoxycholate (Sigma, D6750) and lysed on ice for $10 \mathrm{~min}$. Lysates were centrifuged for

$72910 \mathrm{~min}$ at $10,000 \mathrm{~g}$ at $4^{\circ} \mathrm{C}$. Supernatants were then separated on $10 \%$ or $15 \%$ polyacrylamide

730 SDS-PAGE gels and transferred to polyvinylidene difluoride membranes. Membranes were

731 blocked in TBS-T supplemented with 5\% BSA for $1 \mathrm{~h}$ at room temperature and incubated

732 overnight at $4^{\circ} \mathrm{C}$ with primary antibodies diluted in blocking buffer. They were then

733 incubated with a horseradish peroxidase-conjugated secondary antibody and proteins were

734 detected using enhanced chemiluminescence (GE Healthcare Life Sciences, RPN2209).

735 Densitometry analysis of bands was performed using Image J software.

736

\section{Statistical analysis}

738 Statistical analysis was performed using Graph Pad Prism software. One-way ANOVA

739 followed by Tukey multiple comparison test or unpaired t test were used as indicated in

740 figure legends.

743 Online supplemental material

744 Fig. S1 shows GFP-LC3A and RFP-LAMP1 dynamics during entosis. Fig. S2 shows

745 LysoTracker intensity of phagosomes treated with BafA1 or DPI. Fig. S3 provides additional

746 data on ATG16L1-V-ATPase interaction upon monensin treatment. Video 1 shows GFP-

747 LC3A and Rab7-RFP dynamics during phagocytosis. Video 2 shows GFP-LC3A and

748 LAMP1-RFP dynamics during phagocytosis. 
a

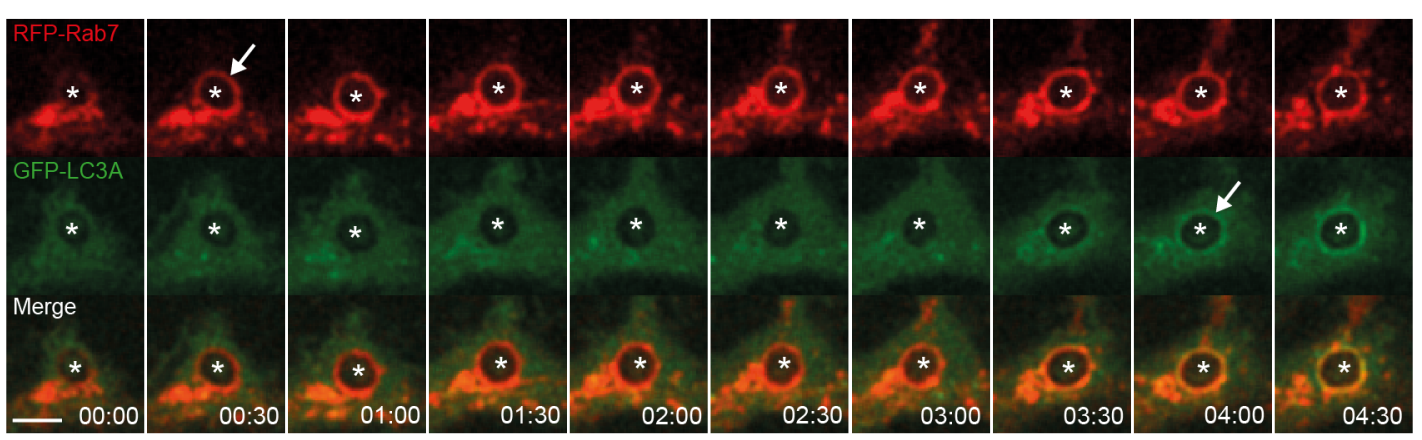

b

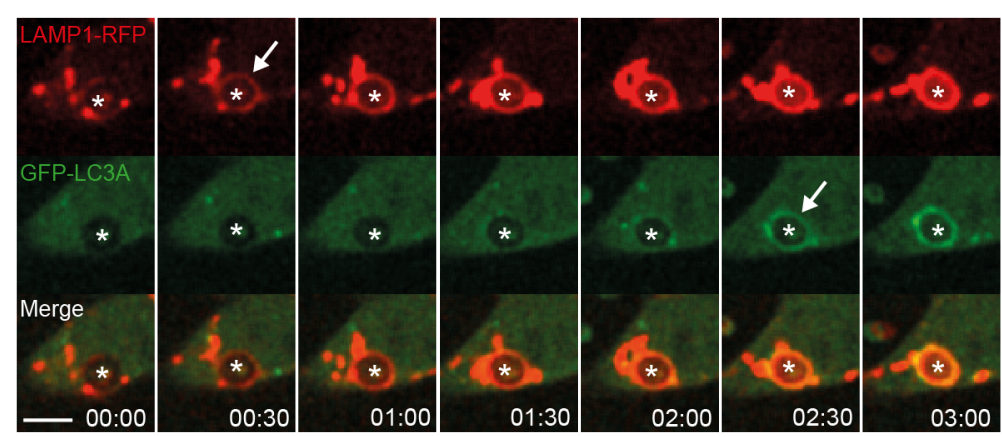

d
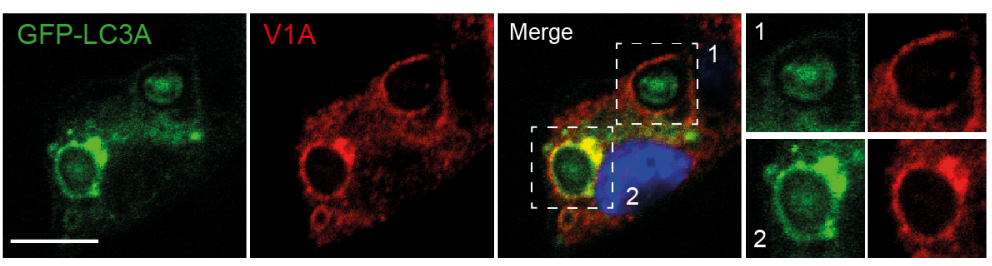

C

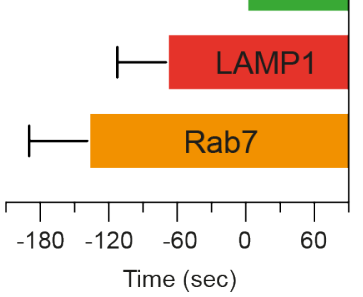

Figure 1

Rab7, LAMP1 and V-ATPase recruit prior to LC3 during LAP. (a and b) Representative timelapse confocal microscopy images of opsonised zymosan (OPZ) phagocytosis in RAW264.7 cells expressing GFP-hLC3A and (a) RFP-Rab7 or (b) LAMP1-RFP. Asterisks denotes phagosomes and arrows mark acquisition of fluorescent markers. Scale bar, $5 \mu \mathrm{m}$, min:sec. (c) Quantification of fluorescent marker acquisition from 10 phagosomes in relation to GFP-hLC3A (time 0). Data represent mean +/- SD. (d) Confocal images of OPZ

761 containing phagosomes stained for GFP-hLC3 and ATP6V1A. Scale bar, $5 \mu \mathrm{m}$. (e) Quantification of phagosome markers from (d). Data represent mean $+/$ - SEM from 3 independent experiments with $>100$ phagosomes analysed per experiment. $* * * * p<0.0001$, $* * \mathrm{p}<0.001$, one-way ANOVA followed by Tukey multiple comparison test. 
a

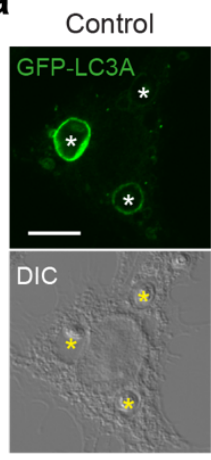

C

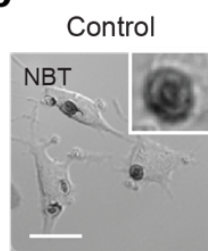

DPI

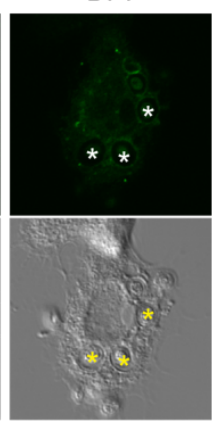

DPI

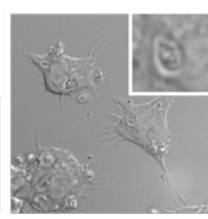

BafA1

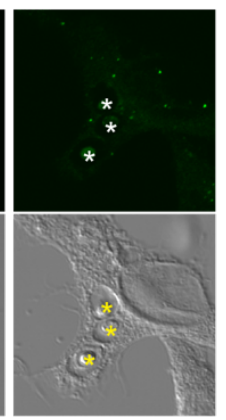

b

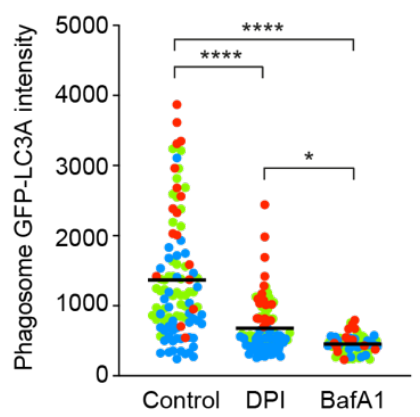

d

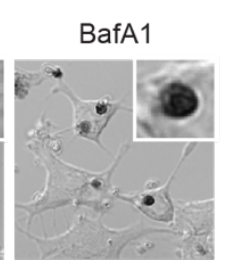

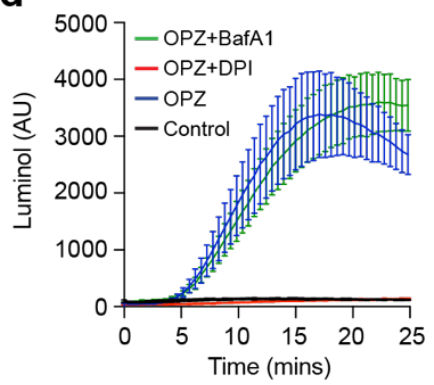

Figure 2

NADPH oxidase and V-ATPase are both required for LAP. (a) Confocal DIC and GFPhLC3A images of OPZ phagocytosis in RAW264.7 cells treated with DPI $(5 \mu \mathrm{M})$ or BafA1 $(100 \mathrm{nM})$. Asterisk denote phagosomes. Scale bar, $5 \mu \mathrm{m}$. (b) Quantification of GFP-hLC3A intensity at phagosomes in cells treated as above. Data represent the mean of individual phagosome measurements from 3 independent experiments (red, green, blue); $* * * * p<0.0001$, ${ }^{*} \mathrm{p}<0.03$, one-way ANOVA followed by Tukey multiple comparison test. (c) Representative confocal DIC images of NBT/formazan deposits in phagosomes from control, DPI and BafA1 treated RAW264.7 cells. Inserts are zoomed in phagosomes. Scale bar, $20 \mu \mathrm{m}$. (d) Luminol measurements of ROS during OPZ phagocytosis in RAW264.7 cells treated with DPI or BafA1. Data represent the mean +/-SEM from 3 independent experiments. 
a
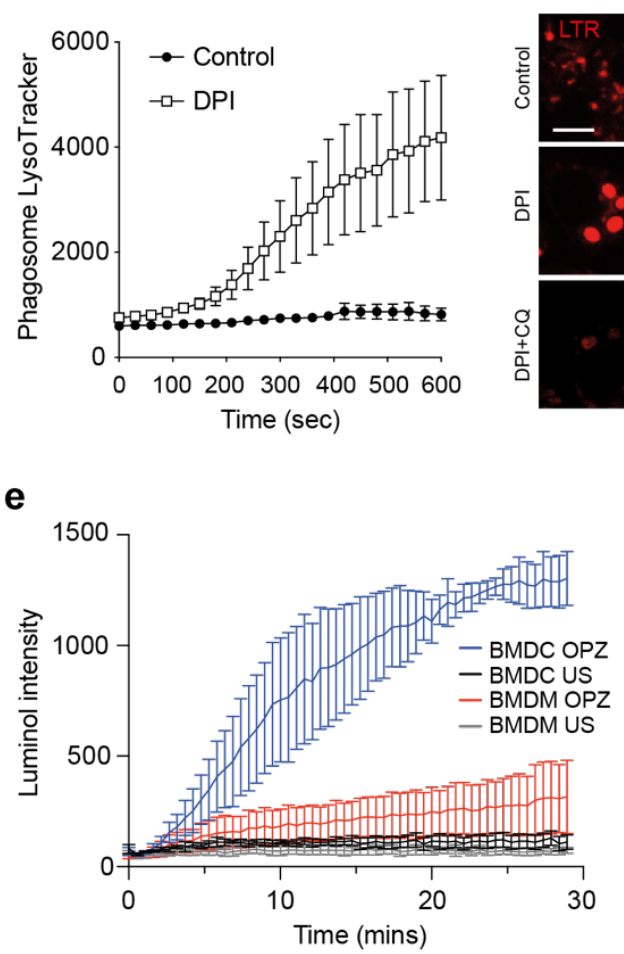

b

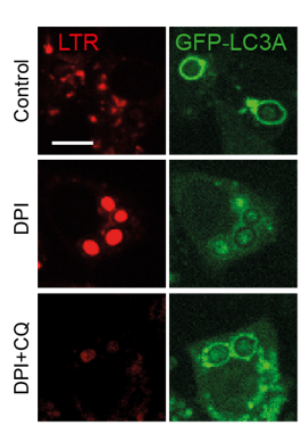

C

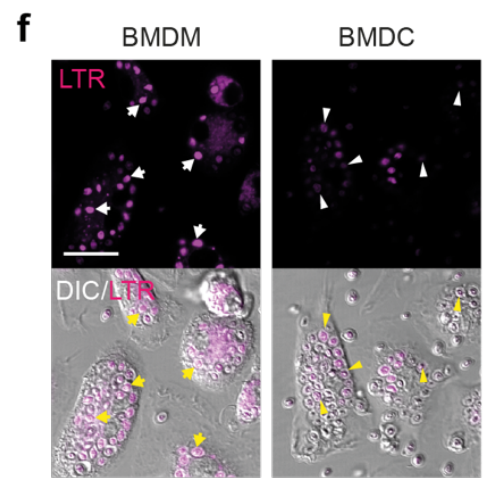

d

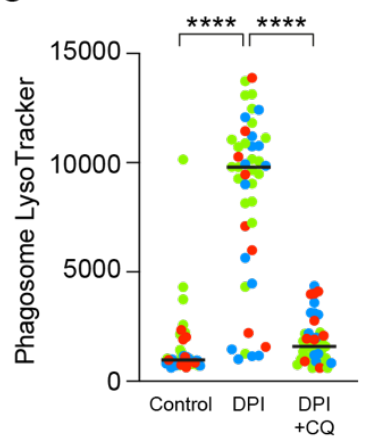

g

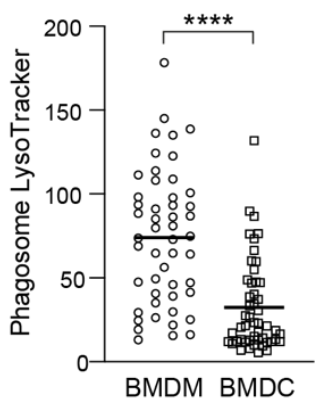

h

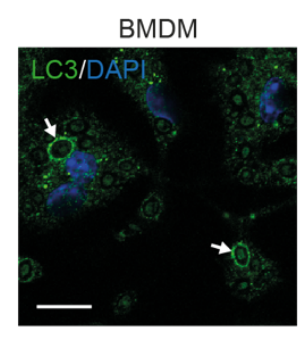

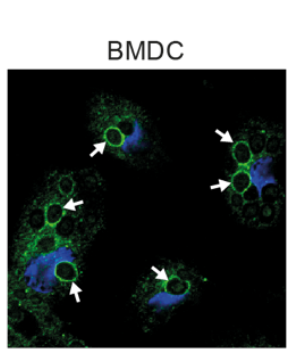

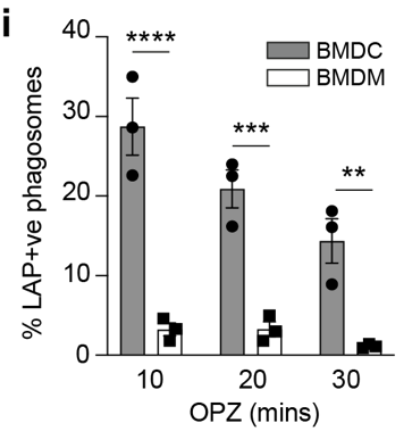

\section{Figure 3}

\section{LAP induction involves elevated ROS and raised $\mathrm{pH}$ in phagosomes. (a) Confocal} microscopy measurement of phagosome LysoTracker intensity over time in RAW264.7 cells stimulated with OPZ +/- DPI $(5 \mu \mathrm{M})$ pre-treatment. Data represent mean +/-SEM of 9 individual phagosomes across multiple independent experiments. (b) RAW264.7 cells were pre-treated with DPI $(5 \mu \mathrm{M})$ or DPI + CQ $(100 \mu \mathrm{M})$ prior to stimulation with OPZ for 25 min. Representative confocal images of LysoTracker and GFP-hLC3A are shown. Scale bar, $5 \mu \mathrm{m}$. (c and d) Quantification of (c) Lysotracker and (d) GFP-hLC3A at phagosomes in cells treated as in (b). Data represent the mean of individual phagosome measurements from 3 independent experiments (red, green, blue). ${ }^{* * * *} p<0.0001$, one-way ANOVA followed by Tukey multiple comparison test. (e) Luminol measurements of ROS during OPZ phagocytosis in primary murine BMDC and BMDM cells. Data represent the mean +/-SEM from 3 independent experiments. (f) Representative confocal images of LysoTracker staining and DIC in BMDC and BMDM cells after stimulation with OPZ for $25 \mathrm{~min}$. Arrows denote phagosomes in BMDMs, arrowheads denote phagosomes in BMDCs. Scale bar, $20 \mu \mathrm{m}$. (g) Quantification of phagosome Lysotracker intensity in cells treated as in (f). Data represent 
bioRxiv preprint doi: https://doi.org/10.1101/2021.05.20.444917; this version posted May 20, 2021. The copyright holder for this preprint

(which was not certified by peer review) is the author/funder, who has granted bioRxiv a license to display the preprint in perpetuity. It is made available under aCC-BY-NC 4.0 International license.

802 the mean of 50 individual phagosomes. $* * * * p<0.0001$, unpaired $t$ test. (h) Representative 803 confocal images of BMDC and BMDM cells stimulated with OPZ for $25 \mathrm{~min}$ and stained for 804 LC3. Arrows denote LC3 positive phagosomes. Scale bar, $10 \mu \mathrm{m}$. (i) Quantification of LC3 805 positive phagosomes from cells stimulated with OPZ for the indicated times. Data represent 806 mean $+/$-SEM from 3 independent experiments. ${ }^{* * *} \mathrm{p}<0.0001,{ }^{* * *} \mathrm{p}<0.0003,{ }^{*} \mathrm{p}<0.003$, 807 two-way ANOVA with multiple comparisons. 
a

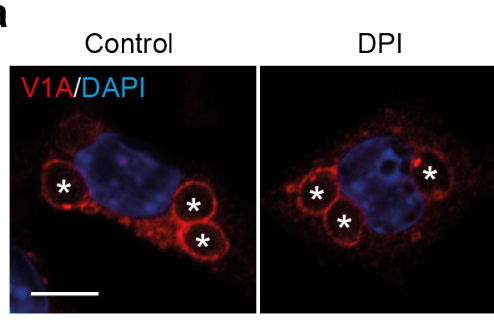

b

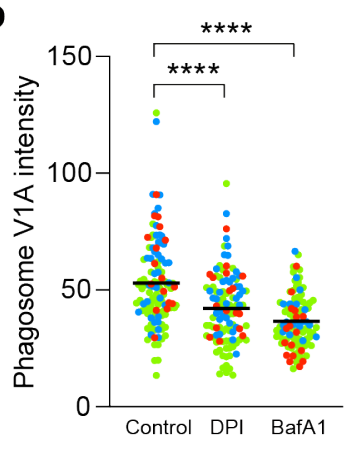

C

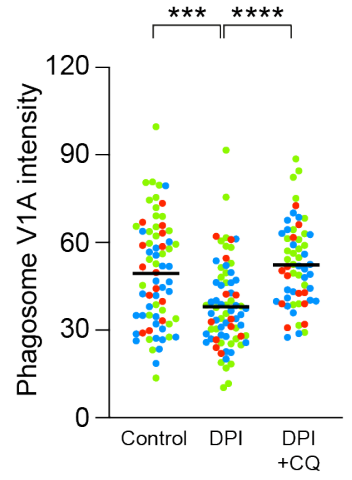

d

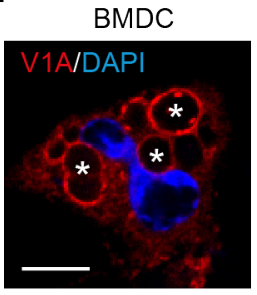

BMDM

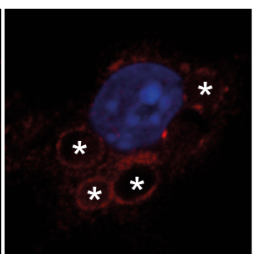

e

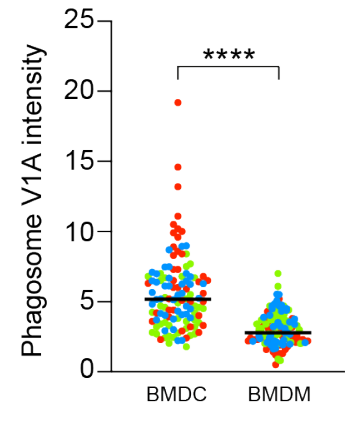

\section{Figure 4}

Modulation of ROS regulates phagosomal V-ATPase recruitment during LAP. (a) phagosomes. Scale bar, $5 \mu \mathrm{m}$. (b) Quantification of ATP6V1A phagosome intensity from cells treated as in (a). Data represent the mean of individual phagosome measurements from 3 independent experiments (red, green, blue). ${ }^{* * * *} \mathrm{p}<0.0001$, one-way ANOVA followed by Tukey multiple comparison test. (c) Quantification of ATP6V1A phagosome intensity from RAW264.7 cells pre-treated with DPI or DPI + CQ. Data represent the mean of individual phagosome measurements from 3 independent experiments (red, green, blue). $* * * * p<0.0001$, one-way ANOVA followed by Tukey multiple comparison test. (d) Representative confocal images of ATP6V1A following 25 min OPZ stimulation of BMDC or BMDM cells. Asterisks denote phagosomes. Scale bar, $5 \mu \mathrm{m}$. (e) Quantification of ATP6V1A phagosome intensity from BMDC and BMDM cells. Data represent the mean of individual phagosome measurements from 3 independent experiments (red, green, blue). $* * * *<<0.0001$, one-way ANOVA followed by Tukey multiple comparison test. 
a

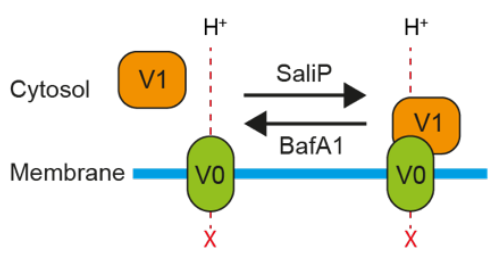

b

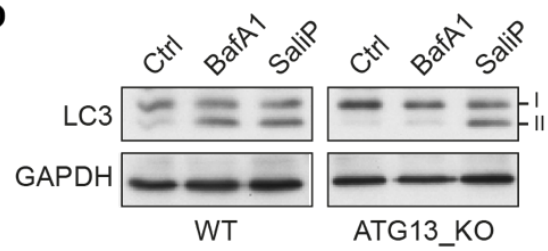

C

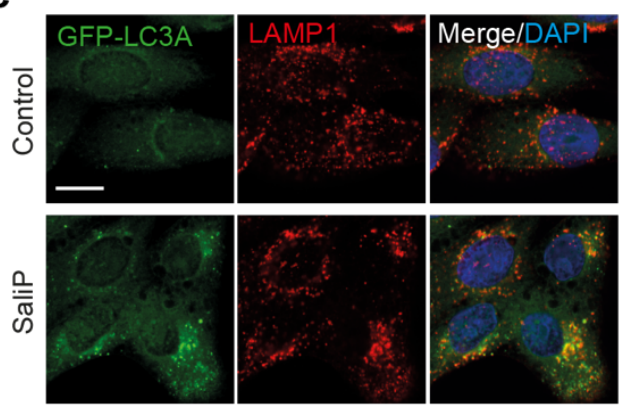

d

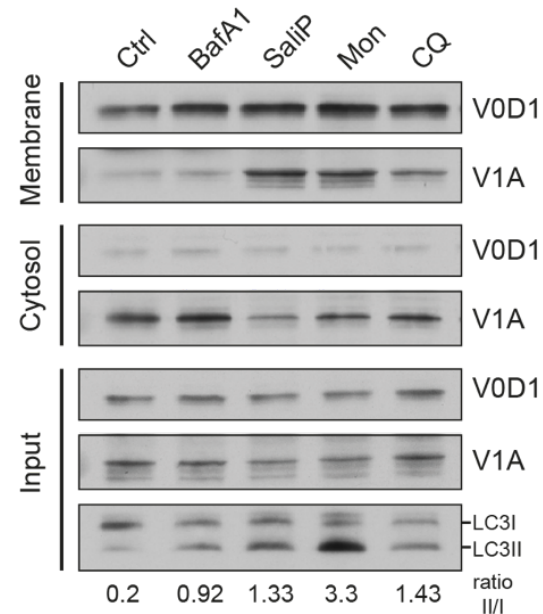

e

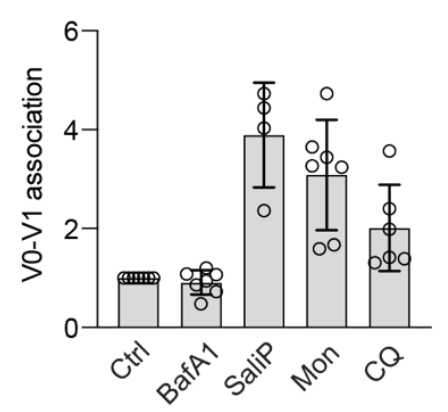

f

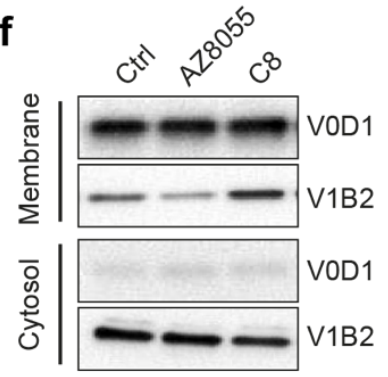

832

833

834

835

836

837

838

839

840

841

842

843

844

845

846

847

848

849

850

851

\section{Figure 5}

\section{V-ATPase V0-V1 engagement is associated with, and sufficient for, non-canonical} autophagy activation. (a) Cartoon showing the differential effects of BafAl and SaliP on V0-V1 association. (b) Wild type and $A T G 13^{-/}$MCF10A cells were treated with BafA1 (100 $\mathrm{nM}$ ) or SaliP $(2.5 \mu \mathrm{M})$ for $1 \mathrm{~h}$. Western blotting was performed to probe for LC3 (I and II forms marked) and GAPDH. (c) Representative confocal images of $A T G 13^{-/-}$MCF 10A cells stained for LAMP1 and GFP-hLC3A following treatment with SaliP $(2.5 \mu \mathrm{M})$ for $1 \mathrm{~h}$. (d) Wild type MCF10A cells were treated with BafA1 $(100 \mathrm{nM})$, SaliP $(2.5 \mu \mathrm{M})$, monensin $(100$ $\mu \mathrm{M})$ or CQ $(100 \mu \mathrm{M})$ for $1 \mathrm{~h}$. Following fractionation, input, membrane and cytosol fractions were probed for ATP6V1A, ATP6V0D1 and LC3 by western blotting. LC3II/LC3I ratios shown below. (e) Quantification of V0-V1 association from experiments as shown in (d). Data represent mean +/-SD from 4-7 independent experiments. (f) HeLa cells were stimulated with either mTor inhibitor AZD8055 $(1 \mu \mathrm{M})$ or TRPML1 agonist C8 $(2 \mu \mathrm{M})$ for $90 \mathrm{~min}$. Following fractionation, membrane and cytosol fractions were probed for ATP6V1B2, ATP6V0D1 by western blotting. 
a

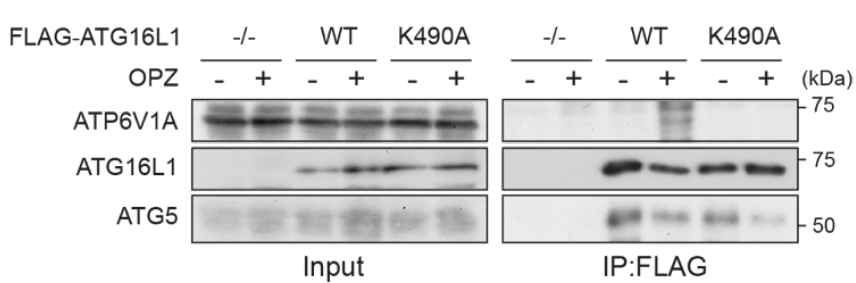

b

b FLAG-ATG16L1
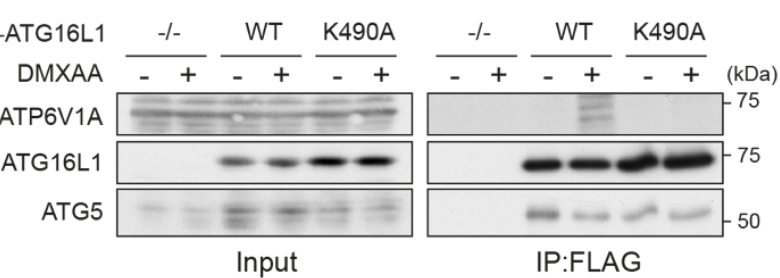

C
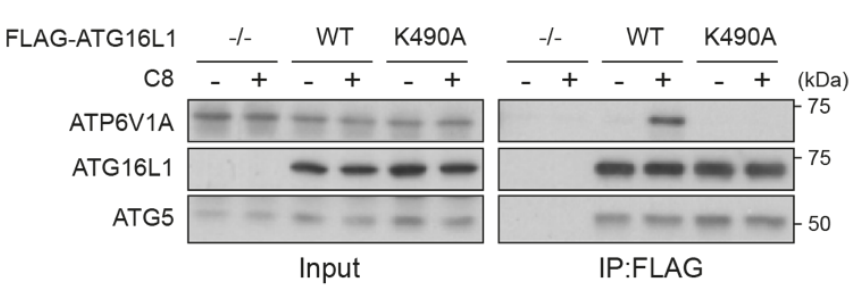

d
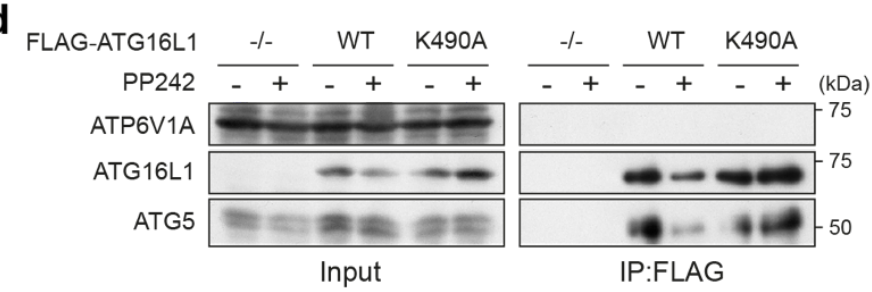

e

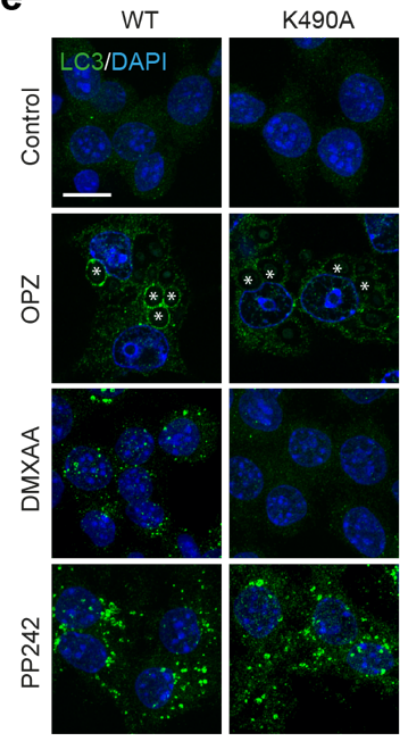

f

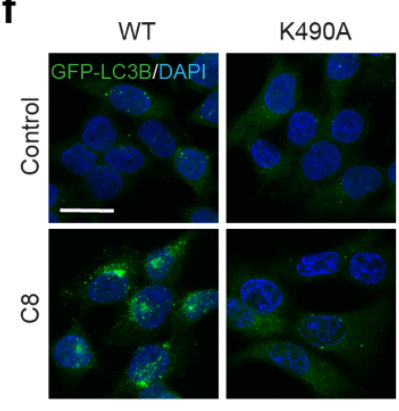

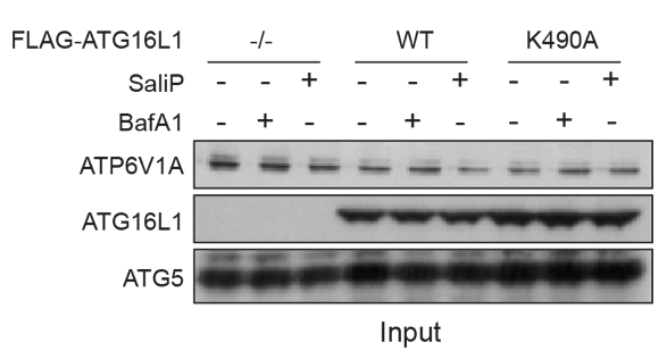

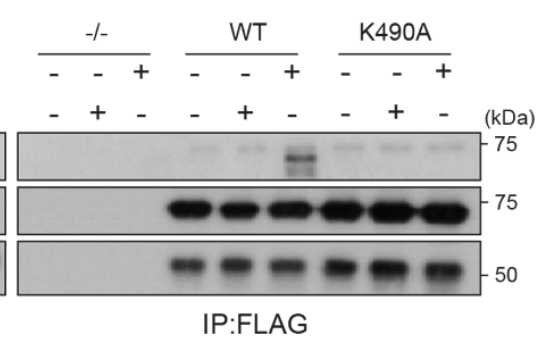

\section{Figure 6}

\section{ATG16L1 interacts with V-ATPase during non-canonical autophagy. (a and b)} $A T G 16 L_{1 /-}$ RAW264.7 cells and those re-expressing Flag-tagged wild type and K490A ATG16L1, were treated with (a) OPZ for 25 min or (b) STING agonist DMXAA $(50 \mu \mathrm{g} / \mathrm{ml})$ for $1 \mathrm{~h}$. Input lysates and Flag immunoprecipitations were probed for ATP6V1A, ATG16L1 and ATG5 by western blotting. (c) ATG16L1/- HCT116 cells and those re-expressing FLAGtagged wild type and K490A ATG16L1, were treated with TRPML1 agonist C8 $(2 \mu \mathrm{M})$ for $30 \mathrm{~min}$. Input lysates and FLAG immunoprecipitations were probed by western blotting as above. (d) $A T G 16 \mathrm{L1}^{-/-}$RAW264.7 cells and those re-expressing FLAG-tagged wild type and K490A ATG16L1, were treated with mTor inhibitor PP242 $(1 \mu \mathrm{M})$ for $1 \mathrm{~h}$. Input lysates and Flag immunoprecipitations were probed by western blotting as above. (e) Confocal images of RAW264.7 cells expressing wild type or K490A ATG16L1 treated with OPZ (25 min), 
bioRxiv preprint doi: https://doi.org/10.1101/2021.05.20.444917; this version posted May 20, 2021 . The copyright holder for this preprint

(which was not certified by peer review) is the author/funder, who has granted bioRxiv a license to display the preprint in perpetuity. It is made available under aCC-BY-NC 4.0 International license.

868 ATG16L1 treated with C8 (30 min). Scale bar, $15 \mu \mathrm{m}$. (g) ATG16L1 $1^{-/}$HCT116 cells and

869 those re-expressing FLAG-tagged wild type and K490A ATG16L1, were treated with BafA1

$870(100 \mathrm{nM})$ or SaliP $(2.5 \mu \mathrm{M})$ for $1 \mathrm{~h}$. Input lysates and FLAG immunoprecipitations were

871 probed by western blotting as above.

872

873

874 
a

GFP-LC3A
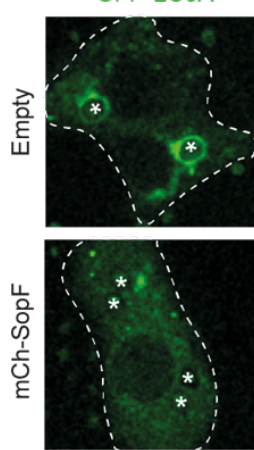

b

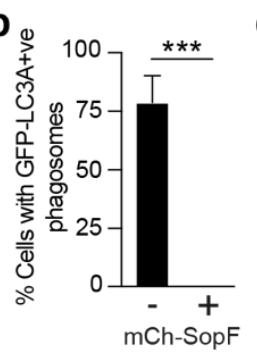

mCherry
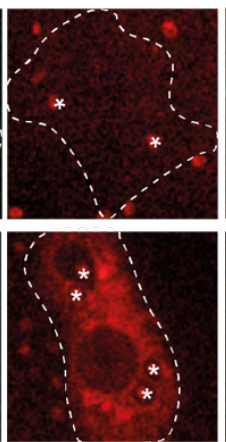

C
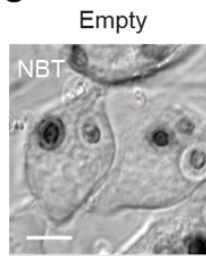

\section{Merge}

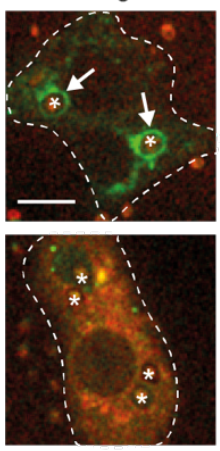

mCh-SopF

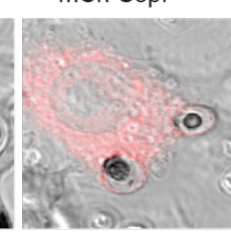

d
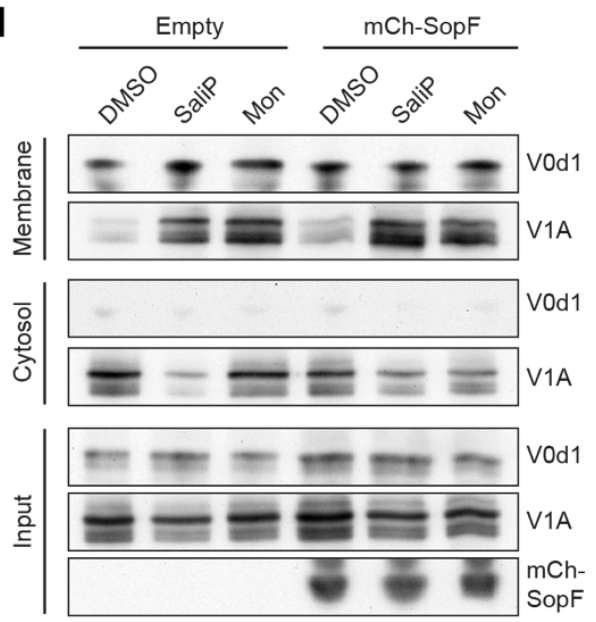

876

877

878

879

880

881

882

883

884

885

886

887

888

889

890

891

892

\section{Figure 7}

The Salmonella effector protein SopF blocks LAP and non-canonical autophagy. (a) Confocal images of GFP-hLC3A expressing RAW264.7 cells transfected with mCherrySopF or empty vector and stimulated with OPZ for $25 \mathrm{~min}$. Asterisks denote phagosomes, arrows mark GFP-LC3A positive phagosomes. Scale bar, $5 \mu \mathrm{m}$. (b) Quantification of the percentage of phagocytosing mCherry-SopF or empty vector expressing cells that contain GFP-hLC3A positive phagosomes following OPZ stimulation. Data represent the mean $+/-$ SEM from 3 independent experiments. ${ }^{* * *} \mathrm{p}<0.0003$, unpaired $t$ test. (c) Representative confocal DIC images of NBT/formazan deposits in phagosomes from empty vector and mCherry-SopF expressing RAW264.7 cells. Scale bar, $5 \mu \mathrm{m}$. (d) Wild type MCF10A cells expressing mCherry-SopF or empty vector were treated with SaliP $(2.5 \mu \mathrm{M})$ or monensin $(100 \mu \mathrm{M})$ for $1 \mathrm{~h}$. Following fractionation, input, membrane and cytosol fractions were probed for ATP6V1A, ATP6V0d1 and mCherry by western blotting. 
a

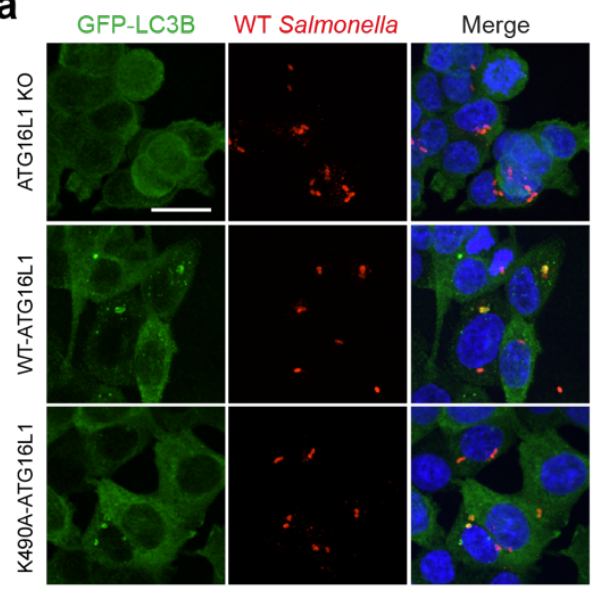

C

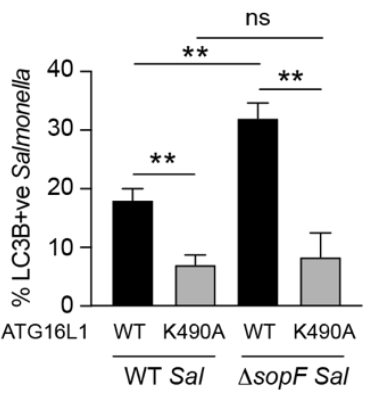

b GFP-LC3B $\triangle$ SopF Salmonella Merge

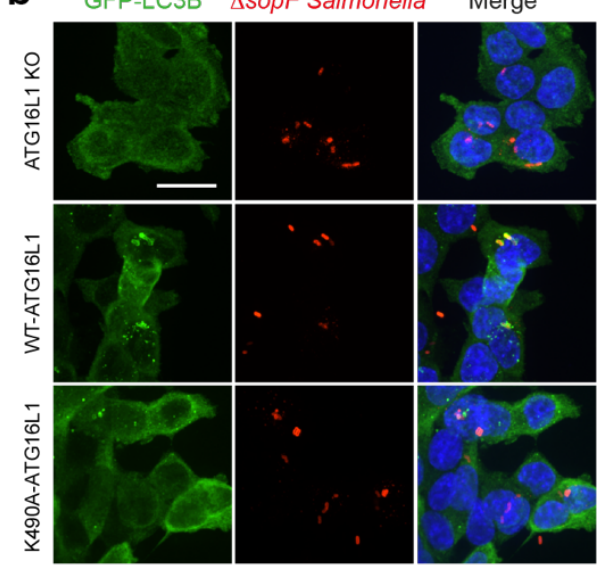

\section{Figure 8}

Non-canonical autophagy contributes to the Salmonella host response. (a and b) ATG $16 \mathrm{~L} \mathrm{1}^{-/}$HCT116 cells and those re-expressing FLAG-tagged wild type and K490A ATG16L1, were infected with either (a) wild type or (b) $\Delta s o p F$ Salmonella for $1 \mathrm{~h}$ and imaged by confocal microscopy. Scale bar, $10 \mu \mathrm{m}$. (c) Quantification of GFP-rLC3B positive

902

903 Salmonella in cells treated as in ( $\mathrm{a}$ and $\mathrm{b}$ ). At least 100 bacteria were counted for each condition. Data represent mean $+/$-SD from 3 independent experiments. ${ }^{* *} \mathrm{p}<0.01$, $* * * * \mathrm{p}<0.0001$, one-way ANOVA followed by Tukey multiple comparison test.

905

906 
910 Model of CASM activation. Stimuli that induce perturbations in endolysosomal ion and $\mathrm{pH}$

911 balance drive V0-V1 engagement that promotes the recruitment of ATG16L1 through its

912 WD40 CTD and results in CASM. SopF modifies V-ATPase to inhibit ATG16L1 interaction

913 while BafA1 interferes with V-ATPase activity, both resulting in inhibition of CASM.

914 During LAP, NOX2 dependent ROS production consumes phagosomal $\mathrm{H}+$ protons, in a DPI

915 sensitive manner, which alters phagosome $\mathrm{pH}$ and drives the SopF-sensitive interaction

916 between V-ATPase and ATG16L1, which then directs ATG8 lipidation to phagosomes. 
bioRxiv preprint doi: https://doi.org/10.1101/2021.05.20.444917; this version posted May 20, 2021. The copyright holder for this preprint

(which was not certified by peer review) is the author/funder, who has granted bioRxiv a license to display the preprint in perpetuity. It is made available under aCC-BY-NC 4.0 International license.

a

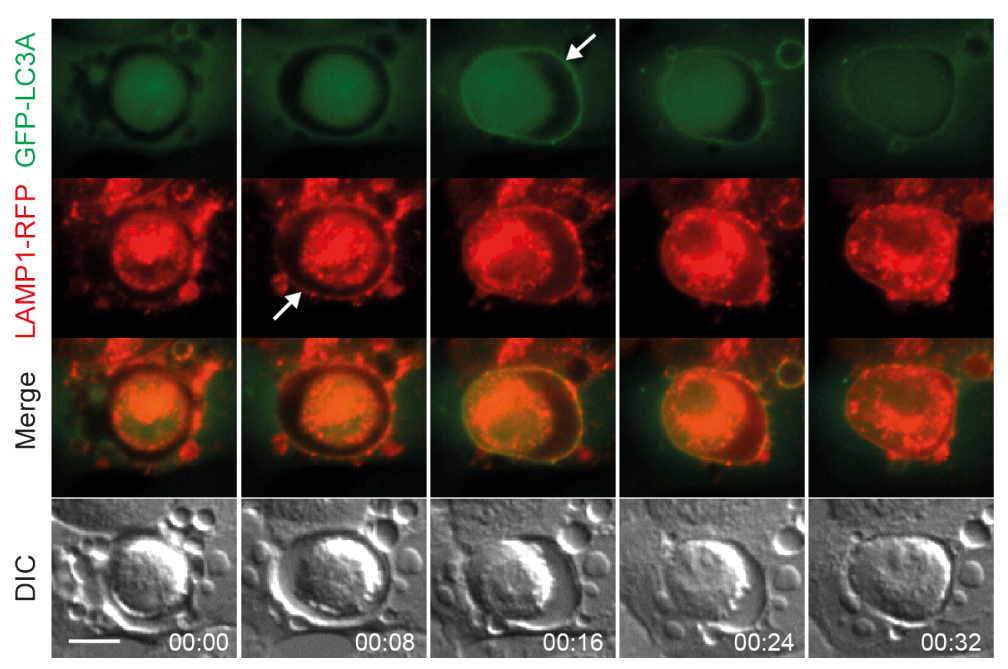

Entosis

b

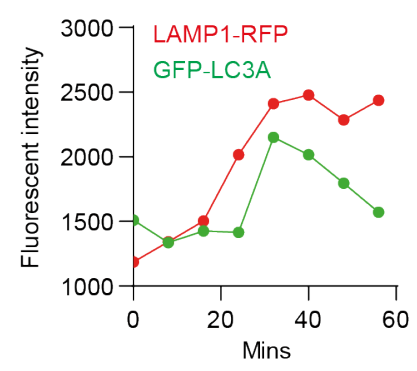

\section{Figure S1}

(a) Representative timelapse widefield microscopy images of entosis in MCF10A cells expressing GFP-hLC3A and LAMP1-RFP. Arrows mark acquisition of fluorescent markers. Scale bar, $15 \mu \mathrm{m}$, min:sec. (b) Analysis of fluorescent marker intensity at the entotic vacuole membrane over time. 


\section{$935 \quad$ Figure S2}

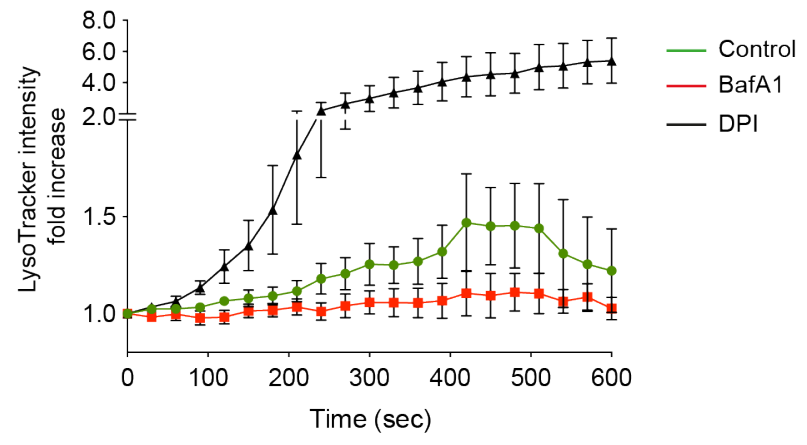

937 Confocal microscopy measurements of phagosome LysoTracker intensity over time in

938 RAW264.7 cells pre-treated with either DPI $(5 \mu \mathrm{M})$ or BafA1 $(100 \mathrm{nM})$ prior to addition of

939 OPZ. Data represent mean values normalised to time $0+/$-SEM of 9 individual phagosomes across multiple independent experiments. 
a

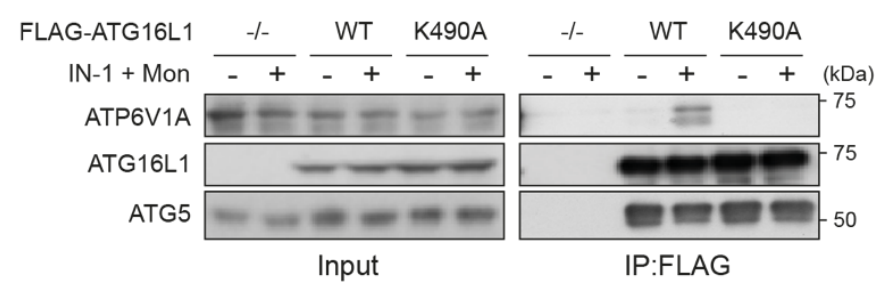

b

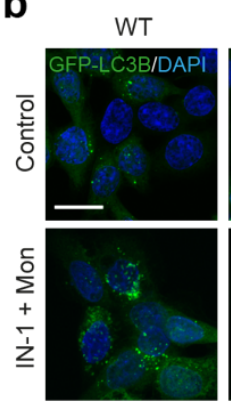

K490A

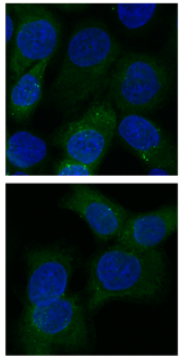

\section{Figure S3}

(a) $A T G 16 \mathrm{L1}^{-/-} \mathrm{HCT} 116$ cells and those re-expressing FLAG-tagged wild type and K490A ATG16L1, were treated with monensin $(100 \mu \mathrm{M})+\mathrm{IN}-1(1 \mu \mathrm{M})$ for $45 \mathrm{~min}$. Input lysates and FLAG immunoprecipitations were probed for ATP6V1A, ATG16L1 and ATG5 by western blotting. (b) Confocal images of GFP-rLC3B HCT116 cells expressing wild type or K490A ATG16L1 treated as in (a). Scale bar, $15 \mu \mathrm{m}$. 
bioRxiv preprint doi: https://doi.org/10.1101/2021.05.20.444917; this version posted May 20,2021 . The copyright holder for this preprint

(which was not certified by peer review) is the author/funder, who has granted bioRxiv a license to display the preprint in perpetuity. It is made available under aCC-BY-NC 4.0 International license.

$957 \quad$ Video 1

958 Timelapse confocal microscopy of opsonised zymosan (OPZ) phagocytosis in RAW264.7

959 cells expressing GFP-hLC3A and RFP-Rab7.

960

961

962

\section{Video 2}

963 Timelapse confocal microscopy of opsonised zymosan (OPZ) phagocytosis in RAW264.7

964 cells expressing GFP-hLC3A and LAMP1-RFP.

965 


\section{References}

Akoumianaki, T., I. Kyrmizi, I. Valsecchi, M.S. Gresnigt, G. Samonis, E. Drakos, D. Boumpas, L. Muszkieta, M.C. Prevost, D.P. Kontoyiannis, T. Chavakis, M.G. Netea, F.L. van de Veerdonk, A.A. Brakhage, J. El-Benna, A. Beauvais, J.P. Latge, and G. Chamilos. 2016. Aspergillus Cell Wall Melanin Blocks LC3-Associated Phagocytosis to Promote Pathogenicity. Cell host \& microbe. 19:79-90.

Cemma, M., S. Grinstein, and J.H. Brumell. 2016. Autophagy proteins are not universally required for phagosome maturation. Autophagy. 12:1440-1446.

Choi, A.M., S.W. Ryter, and B. Levine. 2013. Autophagy in human health and disease. $N$ Engl J Med. 368:651-662.

Collins, M.P., and M. Forgac. 2020. Regulation and function of V-ATPases in physiology and disease. Biochimica et biophysica acta. Biomembranes. 1862:183341.

Cunha, L.D., M. Yang, R. Carter, C. Guy, L. Harris, J.C. Crawford, G. Quarato, E. BoadaRomero, H. Kalkavan, M.D.L. Johnson, S. Natarajan, M.E. Turnis, D. Finkelstein, J.T. Opferman, C. Gawad, and D.R. Green. 2018. LC3-Associated Phagocytosis in Myeloid Cells Promotes Tumor Immune Tolerance. Cell. 175:429-441.e416.

D'Costa, V.M., V. Braun, M. Landekic, R. Shi, A. Proteau, L. McDonald, M. Cygler, S. Grinstein, and J.H. Brumell. 2015. Salmonella Disrupts Host Endocytic Trafficking by SopD2-Mediated Inhibition of Rab7. Cell reports. 12:1508-1518.

De Faveri, F., M. Chvanov, S. Voronina, D. Moore, L. Pollock, L. Haynes, M. Awais, A.J. Beckett, U. Mayer, R. Sutton, D.N. Criddle, I.A. Prior, T. Wileman, and A.V. Tepikin. 2020. LAP-like non-canonical autophagy and evolution of endocytic vacuoles in pancreatic acinar cells. Autophagy. 16:1314-1331.

Dooley, H.C., M. Razi, H.E. Polson, S.E. Girardin, M.I. Wilson, and S.A. Tooze. 2014. WIPI2 links LC3 conjugation with PI3P, autophagosome formation, and pathogen clearance by recruiting Atg12-5-16L1. Mol Cell. 55:238-252.

Durgan, J., A.H. Lystad, K. Sloan, S.R. Carlsson, M.I. Wilson, E. Marcassa, R. Ulferts, J. Webster, A.F. Lopez-Clavijo, M.J. Wakelam, R. Beale, A. Simonsen, D. Oxley, and O. Florey. 2021. Non-canonical autophagy drives alternative ATG8 conjugation to phosphatidylserine. Mol Cell.

Fischer, T.D., C. Wang, B.S. Padman, M. Lazarou, and R.J. Youle. 2020. STING induces LC3B lipidation onto single-membrane vesicles via the V-ATPase and ATG16L1WD40 domain. $J$ Cell Biol. 219.

Fletcher, K., R. Ulferts, E. Jacquin, T. Veith, N. Gammoh, J.M. Arasteh, U. Mayer, S.R. Carding, T. Wileman, R. Beale, and O. Florey. 2018. The WD40 domain of ATG16L1 is required for its non-canonical role in lipidation of LC3 at single membranes. Embo j. 37.

Florey, O., N. Gammoh, S.E. Kim, X. Jiang, and M. Overholtzer. 2015. V-ATPase and osmotic imbalances activate endolysosomal LC3 lipidation. Autophagy. 11:88-99.

Florey, O., S.E. Kim, C.P. Sandoval, C.M. Haynes, and M. Overholtzer. 2011. Autophagy machinery mediates macroendocytic processing and entotic cell death by targeting single membranes. Nat Cell Biol. 13:1335-1343.

Florey, O., and M. Overholtzer. 2012. Autophagy proteins in macroendocytic engulfment. Trends in cell biology. 22:374-380.

Gammoh, N., O. Florey, M. Overholtzer, and X. Jiang. 2013. Interaction between FIP200 and ATG16L1 distinguishes ULK1 complex-dependent and -independent autophagy. Nat Struct Mol Biol. 20:144-149. 
Gluschko, A., M. Herb, K. Wiegmann, O. Krut, W.F. Neiss, O. Utermöhlen, M. Krönke, and M. Schramm. 2018. The $\beta(2)$ Integrin Mac-1 Induces Protective LC3-Associated Phagocytosis of Listeria monocytogenes. Cell host \& microbe. 23:324-337.e325.

Goodwin, J.M., W.G. Walkup, K. Hooper, T. Li, C. Kishi-Itakura, A. Ng, T. Lehmberg, A. Jha, S. Kommineni, K. Fletcher, J. Garcia-Fortanet, Y. Fan, Q. Tang, M. Wei, A. Agrawal, S.R. Budhe, S.R. Rouduri, D. Baird, J. Saunders, J. Kiselar, M.R. Chance, A. Ballabio, B.A. Appleton, J.H. Brumell, O. Florey, and L.O. Murphy. 2021. GABARAP membrane conjugation sequesters the FLCN-FNIP tumor suppressor complex to activate TFEB and lysosomal biogenesis. bioRxiv:2021.2002.2022.432209.

Heckmann, B.L., E. Boada-Romero, L.D. Cunha, J. Magne, and D.R. Green. 2017. LC3Associated Phagocytosis and Inflammation. J Mol Biol. 429:3561-3576.

Heckmann, B.L., B.J.W. Teubner, E. Boada-Romero, B. Tummers, C. Guy, P. Fitzgerald, U. Mayer, S. Carding, S.S. Zakharenko, T. Wileman, and D.R. Green. 2020. Noncanonical function of an autophagy protein prevents spontaneous Alzheimer's disease. Science advances. 6:eabb9036.

Heckmann, B.L., B.J.W. Teubner, B. Tummers, E. Boada-Romero, L. Harris, M. Yang, C.S. Guy, S.S. Zakharenko, and D.R. Green. 2019. LC3-Associated Endocytosis Facilitates beta-Amyloid Clearance and Mitigates Neurodegeneration in Murine Alzheimer's Disease. Cell. 178:536-551.e514.

Henault, J., J. Martinez, J.M. Riggs, J. Tian, P. Mehta, L. Clarke, M. Sasai, E. Latz, M.M. Brinkmann, A. Iwasaki, A.J. Coyle, R. Kolbeck, D.R. Green, and M.A. Sanjuan. 2012. Noncanonical autophagy is required for type I interferon secretion in response to DNA-immune complexes. Immunity. 37:986-997.

Huang, J., V. Canadien, G.Y. Lam, B.E. Steinberg, M.C. Dinauer, M.A. Magalhaes, M. Glogauer, S. Grinstein, and J.H. Brumell. 2009. Activation of antibacterial autophagy by NADPH oxidases. Proceedings of the National Academy of Sciences of the United States of America. 106:6226-6231.

Hubber, A., T. Kubori, C. Coban, T. Matsuzawa, M. Ogawa, T. Kawabata, T. Yoshimori, and H. Nagai. 2017. Bacterial secretion system skews the fate of Legionella-containing vacuoles towards LC3-associated phagocytosis. Scientific reports. 7:44795.

Ichimura, Y., T. Kirisako, T. Takao, Y. Satomi, Y. Shimonishi, N. Ishihara, N. Mizushima, I. Tanida, E. Kominami, M. Ohsumi, T. Noda, and Y. Ohsumi. 2000. A ubiquitin-like system mediates protein lipidation. Nature. 408:488-492.

Jacquin, E., S. Leclerc-Mercier, C. Judon, E. Blanchard, S. Fraitag, and O. Florey. 2017. Pharmacological modulators of autophagy activate a parallel noncanonical pathway driving unconventional LC3 lipidation. Autophagy. 13:854-867.

Kim, J.Y., H. Zhao, J. Martinez, T.A. Doggett, A.V. Kolesnikov, P.H. Tang, Z. Ablonczy, C.C. Chan, Z. Zhou, D.R. Green, and T.A. Ferguson. 2013. Noncanonical autophagy promotes the visual cycle. Cell. 154:365-376.

Kissing, S., C. Hermsen, U. Repnik, C.K. Nesset, K. von Bargen, G. Griffiths, A. Ichihara, B.S. Lee, M. Schwake, J. De Brabander, A. Haas, and P. Saftig. 2015. Vacuolar ATPase in phagosome-lysosome fusion. J Biol Chem. 290:14166-14180.

Kyrmizi, I., H. Ferreira, A. Carvalho, J.A.L. Figueroa, P. Zarmpas, C. Cunha, T. Akoumianaki, K. Stylianou, G.S. Deepe, Jr., G. Samonis, J.F. Lacerda, A. Campos, Jr., D.P. Kontoyiannis, N. Mihalopoulos, K.J. Kwon-Chung, J. El-Benna, I. Valsecchi, A. Beauvais, A.A. Brakhage, N.M. Neves, J.P. Latge, and G. Chamilos. 2018. Calcium sequestration by fungal melanin inhibits calcium-calmodulin signalling to prevent LC3-associated phagocytosis. Nature microbiology. 3:791-803. 
Lafourcade, C., K. Sobo, S. Kieffer-Jaquinod, J. Garin, and F.G. van der Goot. 2008. Regulation of the V-ATPase along the endocytic pathway occurs through reversible subunit association and membrane localization. PLoS One. 3:e2758.

Lau, N., A.L. Haeberle, B.J. O'Keeffe, E.A. Latomanski, J. Celli, H.J. Newton, and L.A. Knodler. 2019. SopF, a phosphoinositide binding effector, promotes the stability of the nascent Salmonella-containing vacuole. PLoS pathogens. 15:e1007959.

Li, S.C., T.T. Diakov, T. Xu, M. Tarsio, W. Zhu, S. Couoh-Cardel, L.S. Weisman, and P.M. Kane. 2014. The signaling lipid $\mathrm{PI}(3,5) \mathrm{P}_{2}$ stabilizes $\mathrm{V}_{1}-\mathrm{V}(\mathrm{o})$ sector interactions and activates the V-ATPase. Mol Biol Cell. 25:1251-1262.

Liberman, R., S. Bond, M.G. Shainheit, M.J. Stadecker, and M. Forgac. 2014. Regulated assembly of vacuolar ATPase is increased during cluster disruption-induced maturation of dendritic cells through a phosphatidylinositol 3-kinase/mTORdependent pathway. J Biol Chem. 289:1355-1363.

Ligeon, L.A., M. Pena-Francesch, L.D. Vanoaica, N.G. Núñez, D. Talwar, T.P. Dick, and C. Münz. 2021. Oxidation inhibits autophagy protein deconjugation from phagosomes to sustain MHC class II restricted antigen presentation. Nat Commun. 12:1508.

Lystad, A.H., S.R. Carlsson, L.R. de la Ballina, K.J. Kauffman, S. Nag, T. Yoshimori, T.J. Melia, and A. Simonsen. 2019. Distinct functions of ATG16L1 isoforms in membrane binding and LC3B lipidation in autophagy-related processes. Nat Cell Biol. 21:372383.

Ma, J., C. Becker, C.A. Lowell, and D.M. Underhill. 2012. Dectin-1-triggered recruitment of light chain 3 protein to phagosomes facilitates major histocompatibility complex class II presentation of fungal-derived antigens. J Biol Chem. 287:34149-34156.

Mantegazza, A.R., A. Savina, M. Vermeulen, L. Pérez, J. Geffner, O. Hermine, S.D. Rosenzweig, F. Faure, and S. Amigorena. 2008. NADPH oxidase controls phagosomal $\mathrm{pH}$ and antigen cross-presentation in human dendritic cells. Blood. 112:4712-4722.

Marshansky, V. 2007. The V-ATPase a2-subunit as a putative endosomal pH-sensor. Biochemical Society transactions. 35:1092-1099.

Martinez, J., J. Almendinger, A. Oberst, R. Ness, C.P. Dillon, P. Fitzgerald, M.O. Hengartner, and D.R. Green. 2011. Microtubule-associated protein 1 light chain 3 alpha (LC3)-associated phagocytosis is required for the efficient clearance of dead cells. Proceedings of the National Academy of Sciences of the United States of America. 108:17396-17401.

Martinez, J., L.D. Cunha, S. Park, M. Yang, Q. Lu, R. Orchard, Q.Z. Li, M. Yan, L. Janke, C. Guy, A. Linkermann, H.W. Virgin, and D.R. Green. 2016. Noncanonical autophagy inhibits the autoinflammatory, lupus-like response to dying cells. Nature. 533:115119.

Martinez, J., R.K. Malireddi, Q. Lu, L.D. Cunha, S. Pelletier, S. Gingras, R. Orchard, J.L. Guan, H. Tan, J. Peng, T.D. Kanneganti, H.W. Virgin, and D.R. Green. 2015. Molecular characterization of LC3-associated phagocytosis reveals distinct roles for Rubicon, NOX2 and autophagy proteins. Nat Cell Biol. 17:893-906.

Masud, S., T.K. Prajsnar, V. Torraca, G.E.M. Lamers, M. Benning, M. Van Der Vaart, and A.H. Meijer. 2019. Macrophages target Salmonella by Lc3-associated phagocytosis in a systemic infection model. Autophagy. 15:796-812.

McGuire, C.M., and M. Forgac. 2018. Glucose starvation increases V-ATPase assembly and activity in mammalian cells through AMP kinase and phosphatidylinositide 3kinase/Akt signaling. J Biol Chem. 293:9113-9123.

Rai, S., M. Arasteh, M. Jefferson, T. Pearson, Y. Wang, W. Zhang, B. Bicsak, D. Divekar, P.P. Powell, R. Naumann, N. Beraza, S.R. Carding, O. Florey, U. Mayer, and T. 
Wileman. 2019. The ATG5-binding and coiled coil domains of ATG16L1 maintain autophagy and tissue homeostasis in mice independently of the WD domain required for LC3-associated phagocytosis. Autophagy. 15:599-612.

Romao, S., N. Gasser, A.C. Becker, B. Guhl, M. Bajagic, D. Vanoaica, U. Ziegler, J. Roesler, J. Dengjel, J. Reichenbach, and C. Münz. 2013. Autophagy proteins stabilize pathogen-containing phagosomes for prolonged MHC II antigen processing. $J$ Cell Biol. 203:757-766.

Sanjuan, M.A., C.P. Dillon, S.W. Tait, S. Moshiach, F. Dorsey, S. Connell, M. Komatsu, K. Tanaka, J.L. Cleveland, S. Withoff, and D.R. Green. 2007. Toll-like receptor signalling in macrophages links the autophagy pathway to phagocytosis. Nature. 450:1253-1257.

Savina, A., C. Jancic, S. Hugues, P. Guermonprez, P. Vargas, I.C. Moura, A.M. LennonDuménil, M.C. Seabra, G. Raposo, and S. Amigorena. 2006. NOX2 controls phagosomal $\mathrm{pH}$ to regulate antigen processing during crosspresentation by dendritic cells. Cell. 126:205-218.

Stransky, L.A., and M. Forgac. 2015. Amino Acid Availability Modulates Vacuolar H+ATPase Assembly. J Biol Chem. 290:27360-27369.

Sun-Wada, G.H., H. Tabata, N. Kawamura, M. Aoyama, and Y. Wada. 2009. Direct recruitment of H+-ATPase from lysosomes for phagosomal acidification. J Cell Sci. 122:2504-2513.

Ulferts, R., E. Marcassa, L. Timimi, L.C. Lee, A. Daley, B. Montaner, S.D. Turner, O. Florey, J.K. Baillie, and R. Beale. 2020. Subtractive CRISPR screen identifies factors involved in non-canonical LC3 lipidation. bioRxiv:2020.2011.2018.388306.

Wang, L., D. Wu, C.V. Robinson, H. Wu, and T.M. Fu. 2020a. Structures of a Complete Human V-ATPase Reveal Mechanisms of Its Assembly. Mol Cell. 80:501-511.e503.

Wang, R., T. Long, A. Hassan, J. Wang, Y. Sun, X.S. Xie, and X. Li. 2020b. Cryo-EM structures of intact V-ATPase from bovine brain. Nat Commun. 11:3921.

Wang, Y., P. Sharma, M. Jefferson, W. Zhang, B. Bone, A. Kipar, D. Bitto, J.L. Coombes, T. Pearson, A. Man, A. Zhekova, Y. Bao, R.A. Tripp, S.R. Carding, Y. Yamauchi, U. Mayer, P.P. Powell, J.P. Stewart, and T. Wileman. 2021. Non-canonical autophagy functions of ATG16L1 in epithelial cells limit lethal infection by influenza A virus. Embo j. 40:e105543.

Westman, J., and S. Grinstein. 2020. Determinants of Phagosomal pH During Host-Pathogen Interactions. Frontiers in cell and developmental biology. 8:624958.

Xie, X.S., D. Padron, X. Liao, J. Wang, M.G. Roth, and J.K. De Brabander. 2004. Salicylihalamide A inhibits the V0 sector of the V-ATPase through a mechanism distinct from bafilomycin A1. J Biol Chem. 279:19755-19763.

Xu, Y., P. Zhou, S. Cheng, Q. Lu, K. Nowak, A.K. Hopp, L. Li, X. Shi, Z. Zhou, W. Gao, D. Li, H. He, X. Liu, J. Ding, M.O. Hottiger, and F. Shao. 2019. A Bacterial Effector Reveals the V-ATPase-ATG16L1 Axis that Initiates Xenophagy. Cell. 178:552566.e520.

Yamamoto, A., Y. Tagawa, T. Yoshimori, Y. Moriyama, R. Masaki, and Y. Tashiro. 1998. Bafilomycin A1 prevents maturation of autophagic vacuoles by inhibiting fusion between autophagosomes and lysosomes in rat hepatoma cell line, H-4-II-E cells. Cell structure and function. 23:33-42. 